Open Access

\title{
Global genetic analyses reveal strong inter-ethnic variability in the loss of activity of the organic cation transporter OCT1
}

Tina Seitz ${ }^{1 \dagger}$, Robert Stalmann ${ }^{1 \dagger}$, Nawar Dalila', Jiayin Chen ${ }^{1}$, Sherin Pojar ${ }^{1}$, Joao N. Dos Santos Pereira ${ }^{1}$, Ralph Krätzner ${ }^{2}$, Jürgen Brockmöller ${ }^{1}$ and Mladen V. Tzvetkov ${ }^{1 *}$

\begin{abstract}
Background: The organic cation transporter OCT1 (SLC22A1) mediates the uptake of vitamin B1, cationic drugs, and xenobiotics into hepatocytes. Nine percent of Caucasians lack or have very low OCT1 activity due to loss-of-function polymorphisms in OCT1 gene. Here we analyzed the global genetic variability in OCT1 to estimate the therapeutic relevance of OCT1 polymorphisms in populations beyond Caucasians and to identify evolutionary patterns of the common loss of OCT1 activity in humans.
\end{abstract}

Methods: We applied massively parallel sequencing to screen for coding polymorphisms in 1,079 unrelated individuals from 53 populations worldwide. The obtained data was combined with the existing 1000 Genomes data comprising an additional 1,092 individuals from 14 populations. The identified OCT1 variants were characterized in vitro regarding their cellular localization and their ability to transport 10 known OCT1 substrates. Both the population genetics data and transport data were used in tandem to generate a world map of loss of OCT1 activity.

Results: We identified 16 amino acid substitutions potentially causing loss of OCT1 function and analyzed them together with five amino acid substitutions that were not expected to affect OCT1 function. The variants constituted 16 major alleles and 14 sub-alleles. Six major alleles showed improper subcellular localization leading to substrate-wide loss in activity. Five major alleles showed correct subcellular localization, but substrate-specific loss of activity. Striking differences were observed in the frequency of loss of OCT1 activity worldwide. While most East Asian and Oceanian individuals had completely functional OCT1, $80 \%$ of native South American Indians lacked functional OCT1 alleles. In East Asia and Oceania the average nucleotide diversity of the loss-of-function variants was much lower than that of the variants that do not affect OCT1 function (ratio of 0.03) and was significantly lower than the theoretically expected heterozygosity (Tajima's $D=-1.64, P<0.01$ ).

Conclusions: Comprehensive genetic analyses showed strong global variations in the frequency of loss of OCT1 activity with selection pressure for maintaining OCT1 activity in East Asia and Oceania. These results not only enable pharmacogenetically-based optimization of drug treatment worldwide, but may help elucidate the functional role of human OCT1.

\footnotetext{
* Correspondence: mtzvetk@gwdg.de

${ }^{\dagger}$ Equal contributors

${ }^{1}$ Institute of Clinical Pharmacology, University Medical Center Göttingen,

Robert-Koch-Str. 40, DE-37075 Göttingen, Germany

Full list of author information is available at the end of the article
}

\section{Biomed Central}

(C) 2015 Seitz et al. This is an Open Access article distributed under the terms of the Creative Commons Attribution License (http://creativecommons.org/licenses/by/4.0), which permits unrestricted use, distribution, and reproduction in any medium, provided the original work is properly credited. The Creative Commons Public Domain Dedication waiver (http:// creativecommons.org/publicdomain/zero/1.0/) applies to the data made available in this article, unless otherwise stated. 


\section{Background}

The polyspecific organic cation transporter OCT1 (SLC22A1) is strongly expressed in human hepatocytes and facilitates uptake of organic cations from the sinusoidal blood. Known substrates of OCT1 are vitamin B1, toxins like 1-methyl-4-phenylpyridinium $\left(\mathrm{MPP}^{+}\right)$and monocrotaline, and clinically relevant drugs like metformin, morphine, tropisetron, tramadol, and others [1-9].

The human OCT1 gene is highly polymorphic. Forty percent of Caucasians carry one and a further $9 \%$ carry two of the following loss-of-function polymorphisms: arginine $_{61}$-to-cysteine (Arg61Cys), cysteine 88 -to-arginine (Cys88Arg), glycine $401^{-t o-s e r i n e ~(G l y 401 S e r), ~ g l y c i n e ~}{ }_{465}{ }^{-}$ to-arginine (Gly465Arg), or a deletion of methinone 420 (Met420del) $[4,10,11]$. Depending on the substrate and zygosity, these individuals completely lack or have substantially reduced OCT1 activity [2-5]. Significant changes in pharmacokinetics and, as a consequence, in clinical effects of several drugs were observed in the carriers of loss-of-function OCT1 polymorphisms [3-5, 12].

The reasons for the frequent loss of OCT1 activity remain elusive. Although a number of endogenous molecules, and recently vitamin $\mathrm{B} 1$, have been found to be substrates of OCT1 $[1,13]$, no major differences in the vitality or fertility were observed between the wild-type and Oct1 knockout mice [14]. However, the liver concentrations of the model substrate tetraethylammonium and the drug metformin were significantly reduced in Oct1 knockout mice [14, 15], suggesting an important role of OCT1 in the hepatic uptake of drugs and xenobiotics with cationic or weak basic structure. Recently, reduced hepatic uptake of vitamin B1 in Oct1 knockout mice was reported to lead to a decreased risk of hepatic steatosis [1]. This decreased risk may improve fitness and provide a survival advantage, although long-term studies in mice and studies in humans to support this are still missing.

Several studies analyzed the genetic variability in OCT1 in detail. Kerb et al. resequenced OCT1 coding and promoter regions in 57 Caucasians and analyzed the polymorphisms they identified in another 190 unrelated Caucasians [10]. They found eight non-synonymous polymorphisms, three of which affected OCT1 activity. Leabman et al. screened for coding genetic variants in OCT1 and 23 other membrane transporters in 247 unrelated individuals: 100 European Americans, 100 African Americans, 30 Asians, 10 Mexicans, and seven Pacific Islanders [16]. Five out of the 15 non-synonymous variants identified by Leabman et al. were shown to strongly decrease OCT1 activity, and three of them completely abolished activity [11]. With a non-synonymous nucleotide diversity of $5.11 \times 10^{-4}$ and a ratio of nonsynonymous to synonymous nucleotide diversity of 0.46 ,
OCT1 had the strongest genetic variability among the organic cation transporters of the SLC22 family [16]. Several studies analyzed the genetic variability of OCT1 in Asians. A total of 230 Japanese individuals were resequenced [17-19] and the identified non-synonymous variants were functionally characterized $[17,20,21]$. The number and the frequency of polymorphisms causing loss of or decreased OCT1 function was much lower in Asians than in Caucasians [17], suggesting the presence of substantial inter-ethnic variability in genetically determined loss of OCT1 activity.

In this study we performed a global-scale population genetics analysis and detailed functional analyses in order to generate a world map of genetically determined loss of OCT1 activity. This map should help to elucidate the potential role of $O C T 1$ polymorphisms in interethnic differences in drug therapy. Furthermore, we were looking for global patterns that may point to factors causing selection pressure for the frequent loss of OCT1 activity. This study expanded the number of individuals and world regions analyzed far beyond the scope of previously published analyses of genetic variability in OCT1. Here we analyzed data from 2,171 unrelated individuals from 67 worldwide populations. Using massively parallel sequencing we screened the OCT1 gene for amino acid substitutions and characterized them for their effects on subcellular localization and transport activity using 10 known OCT1 substrates.

\section{Methods}

\section{Populations studied}

One-thousand and seventy-nine DNA samples from 53 populations worldwide were analyzed in this study: 962 samples from 52 populations worldwide were obtained from the Centre d'Etude du Polymorphisme and belong to the Human Genome Diversity Panel (HGDP-CEPH) [22]. The HGDP-CEPH panel was enriched with 117 DNA samples from healthy Caucasians from Germany [23]. The human DNA samples used in this study were completely anonymized except for geographical region of origin. The collection of the blood specimens that served as sources of DNA was performed in accordance to the Declaration of Helsinki under conditions of informed consent and with institutional review board approvals as described in details elsewhere [22, 23].

In all the key analyses the data obtained from the 1,079 DNA samples was enriched with data from 1,092 samples from the 1000 Genome Project [24]. There was no overlap in the samples between the HGDP-CEPH and 1000 Genome panels. The analyses of the amino acid substitution frequencies, haplotype inferences, analyses of haplotype frequencies, the global analyses of loss of OCT1 activity, and the calculation of the population genetic parameters were performed using the combined 
HGDP-CAPH / 1000 Genomes data (Fig. 1a). Five out of the 14 populations within the 1000 Genomes project represent admixed populations and/or population of unknown geographic origin (the populations CLM, MXL, PUR, CEU, and ASW; Table 1). These populations were not used in the global analyses of loss of OCT1 activity and in the calculations of the population genetic parameters.

\section{Massively parallel re-sequencing}

Eleven regions covering the eleven OCT1 exons and their flanking regions were resequenced using semiconductorbased massively parallel sequencing (Ion Torrent ${ }^{\mathrm{TM}}$, Life Technologies, Darmstadt, Germany). The newly identified, potentially loss-of-function amino acid substitutions were validated by capillary sequencing. Conventional genotyping techniques were used to call individual genotypes of 21 amino acid substitutions. The genotypes of 16 genetic variants (Ser14Phe, Ser29Leu, Arg61Cys, Cys88Arg, Gln97Lys, Pro117Leu, Ser189Leu, Arg206Cys, Gly220Val, Thr245Met, Glu284Lys, Gly401Ser, Gly414Ala,
Met420del, Ile449Thr, and Gly465Arg) were validated by single-base primer-extension reactions $\left(\mathrm{SNaPshot}^{\circ}\right.$, Life Technologies). The genotypes of another five variants (Phe160Leu, Pro341Leu, Arg342His, Met408Val, and Arg488Met) were validated by TaqMan ${ }^{\circ}$-based SNP genotyping assays (Life Technologies). Sex matching was performed to exclude sample swaps and $5 \%$ of the samples were genotyped in duplicate, showing complete concordance in the genotype calls. Arg488Met was genotyped both by $\operatorname{TaqMan}^{\circ}$ and single-base primer-extension. The two methods showed $100 \%$ concordance in the genotype calls. The sequencing and genotyping methods are described in details in the supplementary.

\section{Construction and characterization of OCT1 overexpressing cell lines}

The OCT1-overexpressing HEK293 cells were generated by targeted chromosomal integration using the Flp-In System $^{\text {TM }}$ (Life Technologies, Darmstadt, Germany). The construction and characterization of the cells overexpressing OCT1 alleles *1 (wild type), *3, *4, *5, and *6
A

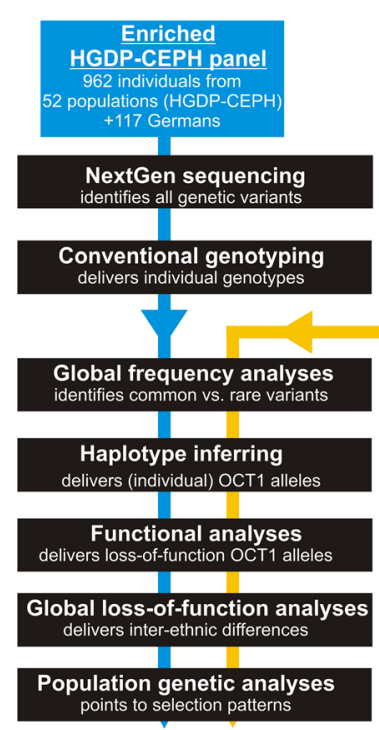

B

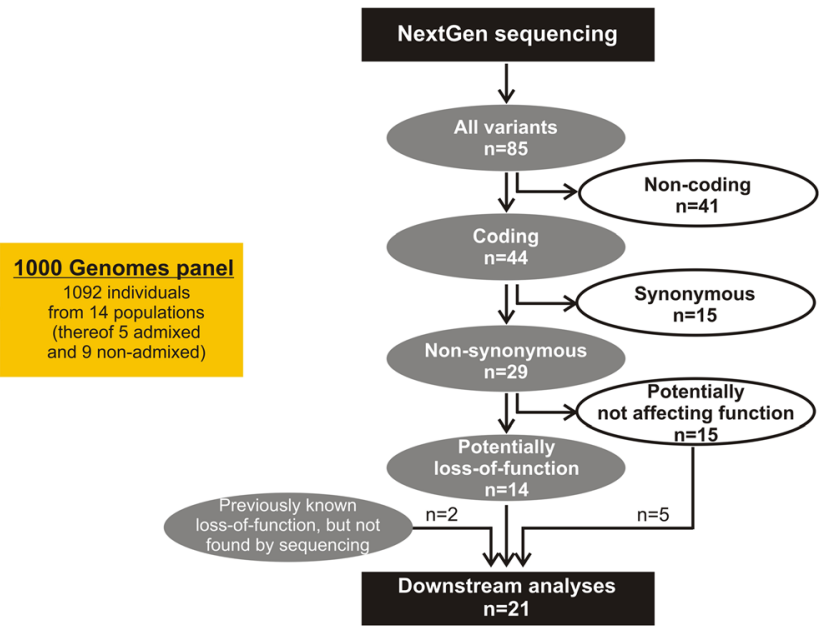

Fig. 1 Schematic representation of the DNA samples and the genetic variants that were analyzed in this study. a A flowchart illustrating which analyses were performed using the enriched HGDP-CEPH panel only and which using both the enriched HGDP-CEPH panel and the 1000 Genomes panel. In the analyses that required unambiguous identification of the geographical origin of the populations (the global frequency analyses, the global loss-of-function, and the population genetic analyses) only the nine non-admixed populations of the 1000 Genomes Project were used together with the 53 populations of the enriched HGDP-CEPH panel. The highly admixed 1000 Genomes populations CLM-1 K, MXL-1 K, PRU-1 K, CEU-1 K, and ASW-1 K were omitted in these analyses. b A flowchart illustrating the process of selection of the 21 genetic variants that were analyzed in detail in this study. The 21 variants were selected from the variants identified by high-throughput sequencing and the variants previously suggested in the literature to cause loss of OCT1 function. In the context of the analysis pipeline presented in panel a, this diagram can be placed between the steps of 'NextGen sequencing' and 'Conventional genotyping'. A detailed list of the non-coding, the synonymous, and the predicted non-deleterious variants is available in Additional file 5 . We regarded variants as potentially deleterious if they were previously reported to cause a more than $50 \%$ decrease in OCT1 activity for at least one substrate; or if they were predicted to be deleterious by at least five of eight software prediction packages used in this study. Details about the prediction software used and the prediction results are given in the text and in Additional files 3 and 6. The variants Gln97Lys and Gly220Val were not identified by sequencing of the enriched HGDP-CEPH panel, but were included in the detailed analyses as they were previously reported in the literature (GIn97Lys, [17]) or in the 1000 Genomes project (Gly220Val) and fulfilled the criteria for deleterious variants 
Table 1 Worldwide distribution of OCT1 alleles

World region Population N chr

\begin{tabular}{|c|c|c|c|c|c|c|c|c|c|c|c|c|c|c|c|c|c|c|c|}
\hline \multirow[t]{2}{*}{ World region } & \multirow[t]{2}{*}{ Population } & \multirow[t]{2}{*}{ N } & \multirow[t]{2}{*}{ chr. } & \multicolumn{16}{|c|}{ Major OCT1 alleles } \\
\hline & & & & ${ }^{* 1} 1$ & ${ }^{*} 2$ & ${ }^{*} 3$ & ${ }^{* 4}$ & *5 & ${ }^{* 6} 6$ & ${ }^{* 7}$ & *8 & *9 & ${ }^{*} 10$ & ${ }^{*} 11$ & ${ }^{* 12}$ & ${ }^{* 13}$ & ${ }^{* 14}$ & *15 & ${ }^{*} 16$ \\
\hline \multirow{9}{*}{$\begin{array}{l}\text { Sub-Saharan } \\
\text { Africa }\end{array}$} & San & 6 & 12 & 91.7 & 0 & 0 & 0 & 0 & 0 & 0 & 8.3 & 0 & 0 & 0 & 0 & 0 & 0 & 0 & 0 \\
\hline & $\begin{array}{l}\text { Mbuti } \\
\text { Pygmy }\end{array}$ & 13 & 26 & 76.9 & 7.7 & 0 & 0 & 0 & 0 & 0 & 15.4 & 0 & 0 & 0 & 0 & 0 & 0 & 0 & 0 \\
\hline & Biaka Pygmy & 26 & 52 & 76.9 & 11.5 & 0 & 0 & 0 & 0 & 9.6 & 1.9 & 0 & 0 & 0 & 0 & 0 & 0 & 0 & 0 \\
\hline & Bantu S.W. & 8 & 16 & 87.5 & 6.3 & 0 & 0 & 0 & 0 & 6.3 & 0 & 0 & 0 & 0 & 0 & 0 & 0 & 0 & 0 \\
\hline & Bantu N.E. & 11 & 22 & 63.6 & 9.1 & 0 & 0 & 0 & 0 & 9.1 & 18.2 & 0 & 0 & 0 & 0 & 0 & 0 & 0 & 0 \\
\hline & $\begin{array}{l}\text { Bantu } \\
\text { (LWK-1 K) }\end{array}$ & 97 & 194 & 88.1 & 4.1 & 0 & 0 & 0 & 0 & 2.6 & 5.1 & 0 & 0 & 0 & 0 & 0 & 0 & 0 & 0 \\
\hline & Mandenka & 22 & 44 & 90.9 & 4.6 & 0 & 0 & 0 & 0 & 2.3 & 2.3 & 0 & 0 & 0 & 0 & 0 & 0 & 0 & 0 \\
\hline & Yoruba & 22 & 44 & 95.5 & 0 & 0 & 0 & 0 & 0 & 2.3 & 2.3 & 0 & 0 & 0 & 0 & 0 & 0 & 0 & 0 \\
\hline & $\begin{array}{l}\text { Yoruba } \\
\text { (YRI-1 K) }\end{array}$ & 88 & 176 & 90.3 & 2.3 & 0 & 0 & 0 & 0 & 1.7 & 5.7 & 0 & 0 & 0 & 0 & 0 & 0 & 0 & 0 \\
\hline $\begin{array}{l}\text { Sub-Saharan } \\
\text { Africa mean }\end{array}$ & & 293 & 586 & 84.6 & 5.1 & 0 & 0 & 0 & 0 & 3.8 & 6.6 & & 0 & 0 & 0 & 0 & 0 & 0 & 0 \\
\hline (range) & & & & (63.6-95.5) & $(0.0-11.5)$ & & & & & $(0.0-9.6)$ & $(0.0-18.2)$ & & & & & & & & \\
\hline \multirow{4}{*}{$\begin{array}{l}\text { North Africa } \\
\text { and Middle } \\
\text { East }\end{array}$} & Mozabite & 29 & 58 & 82.8 & 12.1 & 0 & 1.72 & 0 & 1.72 & 1.72 & 0 & 0 & 0 & 0 & 0 & 0 & 0 & 0 & 0 \\
\hline & Bedouin & 46 & 92 & 72.8 & 9.8 & 10.9 & 0 & 3.26 & 0 & 2.17 & 1.1 & 0 & 0 & 0 & 0 & 0 & 0 & 0 & 0 \\
\hline & Palestinian & 45 & 90 & 78.9 & 13.3 & 3.33 & 0 & 0 & 2.22 & 2.22 & 0 & 0 & 0 & 0 & 0 & 0 & 0 & 0 & 0 \\
\hline & Druze & 42 & 84 & 77.4 & 17.9 & 4.76 & 0 & 0 & 0 & 0 & 0 & 0 & 0 & 0 & 0 & 0 & 0 & 0 & 0 \\
\hline $\begin{array}{l}\text { North Africa } \\
\text { and Middle } \\
\text { East mean }\end{array}$ & & 162 & 324 & 78.2 & 13.3 & 4.7 & 0.4 & 0.8 & 1.0 & 1.5 & 0.3 & 0 & 0 & 0 & 0 & 0 & 0 & 0 & 0 \\
\hline (range) & & & & (73.9-82.8) & $(9.8-17.9)$ & $(0.0-10.9)$ & $(0.0-1.7)$ & $(0.0-3.3)$ & $(0.0-2.2)$ & $(0.0-2.2)$ & $(0.0-1.12)$ & & & & & & & & \\
\hline \multirow[t]{10}{*}{ Europe } & Tuscan & 8 & 16 & 87.5 & 12.5 & 0 & 0 & 0 & 0 & 0 & 0 & 0 & 0 & 0 & 0 & 0 & 0 & 0 & 0 \\
\hline & $\begin{array}{l}\text { Tuscan } \\
\text { (TSI-1 K) }\end{array}$ & 98 & 196 & 71.9 & 18.9 & 5.6 & 2.55 & 0.51 & 0 & 0 & 0.5 & 0 & 0.5 & 0 & 0 & 0 & 0 & 0 & 0 \\
\hline & Sardinian & 28 & 56 & 69.6 & 21.4 & 1.8 & 5.36 & 0 & 1.79 & 0 & 0 & 0 & 0 & 0 & 0 & 0 & 0 & 0 & 0 \\
\hline & North Italian & 14 & 28 & 89.3 & 7.14 & 3.6 & 0 & 0 & 0 & 0 & 0 & 0 & 0 & 0 & 0 & 0 & 0 & 0 & 0 \\
\hline & $\begin{array}{l}\text { Iberian } \\
\text { (BBS-1 K) }\end{array}$ & 14 & 28 & 75.0 & 14.3 & 3.6 & 7.14 & 0 & 0 & 0 & 0 & 0 & 0 & 0 & 0 & 0 & 0 & 0 & 0 \\
\hline & $\begin{array}{l}\text { French } \\
\text { Basque }\end{array}$ & 24 & 48 & 72.9 & 18.8 & 4.2 & 4.17 & 0 & 0 & 0 & 0 & 0 & 0 & 0 & 0 & 0 & 0 & 0 & 0 \\
\hline & French & 28 & 56 & 62.5 & 21.4 & 7.14 & 1.79 & 5.36 & 1.79 & 0 & 0 & 0 & 0 & 0 & 0 & 0 & 0 & 0 & 0 \\
\hline & $\begin{array}{l}\text { British } \\
\text { (GBR-1 K) }\end{array}$ & 89 & 178 & 72.5 & 16.3 & 5.06 & 2.25 & 3.37 & 0.56 & 0 & 0 & 0 & 0 & 0 & 0 & 0 & 0 & 0 & 0 \\
\hline & Orcadian & 15 & 30 & 80.0 & 13.3 & 6.67 & 0 & 0 & 0 & 0 & 0 & 0 & 0 & 0 & 0 & 0 & 0 & 0 & 0 \\
\hline & German & 117 & 234 & 71.4 & 15.8 & 8.12 & 2.14 & 2.56 & 0 & 0 & 0 & 0 & 0 & 0 & 0 & 0 & 0 & 0 & 0 \\
\hline
\end{tabular}


Table 1 Worldwide distribution of OCT1 alleles (Continued)

\begin{tabular}{|c|c|c|c|c|c|c|c|c|c|c|c|c|c|c|c|c|c|c|c|}
\hline & $\begin{array}{l}\text { Finn } \\
(\text { FIN-1 K) }\end{array}$ & 93 & 186 & 78.5 & 13.4 & 5.38 & 1.61 & 1.08 & 0 & 0 & 0 & 0 & 0 & 0 & 0 & 0 & 0 & 0 & 0 \\
\hline & Adygei & 17 & 34 & 70.6 & 17.6 & 5.88 & 0 & 2.94 & 0 & 0 & 0 & 0 & 2.94 & 0 & 0 & 0 & 0 & 0 & 0 \\
\hline & Russian & 25 & 50 & 66.0 & 10.0 & 10.0 & 6.00 & 8.00 & 0 & 0 & 0 & 0 & 0 & 0 & 0 & 0 & 0 & 0 & 0 \\
\hline Europe mean & & 570 & 1,140 & 74.4 & 15.5 & 5.1 & 2.5 & 1.8 & 0.3 & 0 & 0.04 & & 0.3 & 0 & 0 & 0 & 0 & 0 & 0 \\
\hline (range) & & & & $(62.5-89.3)$ & $(7.1-21.4)$ & $(0.0-10.0)$ & $(0.0-7.1)$ & $(0.0-8.0)$ & $(0.0-1.8)$ & & $(0.0-0.5)$ & & $(0.0-2.94)$ & & & & & & \\
\hline \multirow[t]{10}{*}{ Central Asia } & Balochi & 24 & 48 & 66.7 & 27.1 & 6.25 & 0 & 0 & 0 & 0 & 4.2 & 0 & 0 & 0 & 0 & 0 & 0 & 0 & 0 \\
\hline & Brahui & 25 & 50 & 62.0 & 26.0 & 6.00 & 0 & 6 & 0 & 0 & 0 & 0 & 0 & 0 & 0 & 2 & 0 & 0 & 0 \\
\hline & Makrani & 25 & 50 & 84.0 & 16.0 & 0 & 0 & 0 & 0 & 0 & 0 & 0 & 0 & 0 & 0 & 0 & 0 & 0 & 0 \\
\hline & Sindhi & 24 & 48 & 83.3 & 12.5 & 0 & 0 & 0 & 0 & 4.17 & 0 & 0 & 0 & 0 & 0 & 0 & 0 & 0 & 0 \\
\hline & Pathan & 25 & 50 & 72 & 26 & 2 & 0 & 0 & 0 & 0 & 0 & 0 & 0 & 0 & 0 & 0 & 0 & 0 & 0 \\
\hline & Kalash & 23 & 46 & 73.9 & 19.6 & 6.52 & 0 & 0 & 0 & 0 & 0 & 0 & 0 & 0 & 0 & 2.17 & 0 & 0 & 0 \\
\hline & Burusho & 25 & 50 & 84 & 6 & 6 & 0 & 4.00 & 0 & 0 & 0 & 0 & 0 & 0 & 0 & 0 & 0 & 0 & 0 \\
\hline & Hazara & 23 & 46 & 89.1 & 4.35 & 2.17 & 0 & 0 & 2.17 & 0 & 0 & 0 & 0 & 0 & 0 & 0 & 2.17 & 0 & 0 \\
\hline & Uygur & 10 & 20 & 80 & 15 & 5 & 0 & 0 & 0 & 0 & 0 & 0 & 0 & 0 & 0 & 0 & 0 & 0 & 0 \\
\hline & Yakut & 25 & 50 & 92.0 & 0 & 4.00 & 0 & 0 & 0 & 0 & 0 & 4.00 & 0 & 0 & 0 & 0 & 0 & 0 & 0 \\
\hline $\begin{array}{l}\text { Central Asia } \\
\text { mean }\end{array}$ & & 229 & 458 & 78.7 & 14.8 & 3.8 & 0 & 1.0 & 0.2 & 0.4 & 0.42 & 0.4 & 0 & 0 & 0 & 0.4 & 0.2 & 0 & 0 \\
\hline (range) & & & & $(62.0-92.0)$ & $(0.0-27.1)$ & $(0.0-6.5)$ & & $(0.0-6.0)$ & $(0.0-2.2)$ & $(0.0-4.2)$ & $(0.0-4.2)$ & $(0.0-4.0)$ & & & & $(0.0-2.2)$ & $(0.0-2.2)$ & & \\
\hline \multirow{16}{*}{$\begin{array}{l}\text { East Asia and } \\
\text { Oceania }\end{array}$} & Dai & 10 & 20 & 100 & 0 & 0 & 0 & 0 & 0 & 0 & 0 & 0 & 0 & 0 & 0 & 0 & 0 & 0 & 0 \\
\hline & She & 10 & 20 & 90.0 & 0 & 0 & 0 & 0 & 0 & 0 & 0 & 0 & 0 & 0 & 10.0 & 0 & 0 & 0 & 0 \\
\hline & Tujia & 10 & 20 & 100 & 0 & 0 & 0 & 0 & 0 & 0 & 0 & 0 & 0 & 0 & 0 & 0 & 0 & 0 & 0 \\
\hline & Yizu & 10 & 20 & 100 & 0 & 0 & 0 & 0 & 0 & 0 & 0 & 0 & 0 & 0 & 0 & 0 & 0 & 0 & 0 \\
\hline & Naxi & 9 & 18 & 94.4 & 0 & 0 & 0 & 0 & 0 & 0 & 0 & 0 & 0 & 5.56 & 0 & 0 & 0 & 0 & 0 \\
\hline & $\mathrm{Tu}$ & 10 & 20 & 90.0 & 0 & 0 & 0 & 0 & 0 & 0 & 0 & 0 & 0 & 0 & 0 & 0 & 0 & 5.0 & 0 \\
\hline & Xibo & 9 & 18 & 100 & 0 & 0 & 0 & 0 & 0 & 0 & 0 & 0 & 0 & 0 & 0 & 0 & 0 & 0 & 0 \\
\hline & Mongola & 10 & 20 & 85.0 & 10.0 & 0 & 0 & 0 & 0 & 0 & 0 & 5.00 & 0 & 0 & 0 & 0 & 0 & 0 & 0 \\
\hline & Hezhen & 9 & 18 & 100 & 0 & 0 & 0 & 0 & 0 & 0 & 0 & 0 & 0 & 0 & 0 & 0 & 0 & 0 & 0 \\
\hline & Daur & 10 & 20 & 100 & 0 & 0 & 0 & 0 & 0 & 0 & 0 & 0 & 0 & 0 & 0 & 0 & 0 & 0 & 0 \\
\hline & Oroqen & 9 & 18 & 100 & 0 & 0 & 0 & 0 & 0 & 0 & 0 & 0 & 0 & 0 & 0 & 0 & 0 & 0 & 0 \\
\hline & Han & 45 & 90 & 100 & 0 & 0 & 0 & 0 & 0 & 0 & 0 & 0 & 0 & 0 & 0 & 0 & 0 & 0 & 0 \\
\hline & $\begin{array}{l}\text { Han Beijing } \\
\text { (CHB-1 K) }\end{array}$ & 97 & 194 & 98.5 & 0.52 & 0 & 0 & 0 & 0 & 0 & 0 & 0.52 & 0 & 0.52 & 0 & 0 & 0 & 0 & 0 \\
\hline & $\begin{array}{l}\text { Han South } \\
\text { (CHS-1 K) }\end{array}$ & 100 & 200 & 100 & 0 & 0 & 0 & 0 & 0 & 0 & 0 & 0 & 0 & 0 & 0 & 0 & 0 & 0 & 0 \\
\hline & Lahu & 8 & 16 & 100 & 0 & 0 & 0 & 0 & 0 & 0 & 0 & 0 & 0 & 0 & 0 & 0 & 0 & 0 & 0 \\
\hline & Miaozu & 10 & 20 & 100 & 0 & 0 & 0 & 0 & 0 & 0 & 0 & 0 & 0 & 0 & 0 & 0 & 0 & 0 & 0 \\
\hline
\end{tabular}


Table 1 Worldwide distribution of OCT1 alleles (Continued)

\begin{tabular}{|c|c|c|c|c|c|c|c|c|c|c|c|c|c|c|c|c|c|c|c|}
\hline & Cambodian & 10 & 20 & 95.0 & 5.0 & 0 & 0 & 0 & 0 & 0 & 0 & 0 & 0 & 0 & 0 & 0 & 0 & 0 & 0 \\
\hline & Japanese & 30 & 60 & 100 & 0 & 0 & 0 & 0 & 0 & 0 & 0 & 0 & 0 & 0 & 0 & 0 & 0 & 0 & 0 \\
\hline & $\begin{array}{l}\text { Japanese } \\
\text { (JPT-1 K) }\end{array}$ & 89 & 178 & 100 & 0 & 0 & 0 & 0 & 0 & 0 & 0 & 0 & 0 & 0 & 0 & 0 & 0 & 0 & 0 \\
\hline & Melanesian & 14 & 28 & 100 & 0 & 0 & 0 & 0 & 0 & 0 & 0 & 0 & 0 & 0 & 0 & 0 & 0 & 0 & 0 \\
\hline & Papuan & 17 & 34 & 100 & 0 & 0 & 0 & 0 & 0 & 0 & 0 & 0 & 0 & 0 & 0 & 0 & 0 & 0 & 0 \\
\hline $\begin{array}{l}\text { East Asia and } \\
\text { Oceania mean }\end{array}$ & & 526 & 1,052 & 97.8 & 0.7 & 0 & 0 & 0 & 0 & 0 & 0 & 0.3 & 0 & 0.3 & 0.5 & 0 & 0 & 0.2 & 0.2 \\
\hline (range) & & & & $(85.0-100.0)$ & $(0.0-10.0)$ & & & & & & & $(0.0-5.0)$ & & $(0.0-5.6)$ & $(0.0-10.0)$ & & & $(0.0-5.0)$ & $(0.0-5.0)$ \\
\hline \multirow[t]{5}{*}{ America } & Colombian & 7 & 14 & 57.1 & 42.9 & 0 & 0 & 0 & 0 & 0 & 0 & 0 & 0 & 0 & 0 & 0 & 0 & 0 & 0 \\
\hline & Karitiana & 14 & 28 & 39.3 & 60.7 & 0 & 0 & 0 & 0 & 0 & 0 & 0 & 0 & 0 & 0 & 0 & 0 & 0 & 0 \\
\hline & Maya & 21 & 42 & 66.7 & 33.3 & 0 & 0 & 0 & 0 & 0 & 0 & 0 & 0 & 0 & 0 & 0 & 0 & 0 & 0 \\
\hline & Pima & 14 & 28 & 82.1 & 17.9 & 0 & 0 & 0 & 0 & 0 & 0 & 0 & 0 & 0 & 0 & 0 & 0 & 0 & 0 \\
\hline & Surui & 8 & 16 & 6.25 & 93.8 & 0 & 0 & 0 & 0 & 0 & 0 & 0 & 0 & 0 & 0 & 0 & 0 & 0 & 0 \\
\hline America mean & & 64 & 128 & 50.3 & 49.7 & 0 & 0 & 0 & 0 & 0 & 0 & 0 & 0 & 0 & 0 & 0 & 0 & 0 & 0 \\
\hline (range) & & & & $(6.3-82.1)$ & $(17.9-93.7)$ & & & & & & & & & & & & & & \\
\hline \multirow[t]{5}{*}{$\begin{array}{l}\text { Admixed } \\
\text { populations }\end{array}$} & $\begin{array}{l}\text { Colombian } \\
\text { (CLM-1 K) }\end{array}$ & 60 & 120 & 65.8 & 29.2 & 3.33 & 0.83 & 0.83 & 0 & 0 & 0 & 0 & 0 & 0 & 0 & 0 & 0 & 0 & 0 \\
\hline & $\begin{array}{l}\text { Mexican } \\
(\text { MXL-1 K) }\end{array}$ & 66 & 132 & 59.1 & 32.6 & 4.55 & 0 & 3.03 & 0 & 0.76 & 0 & 0 & 0 & 0 & 0 & 0 & 0 & 0 & 0 \\
\hline & $\begin{array}{l}\text { Puerto Rican } \\
\text { (PUR-1 K) }\end{array}$ & 55 & 110 & 76.4 & 20.0 & 0.91 & 0 & 1.82 & 0 & 0 & 0 & 0 & 0.91 & 0 & 0 & 0 & 0 & 0 & 0 \\
\hline & $\begin{array}{l}\text { Caucasians } \\
\text { (CEU-1 K) }\end{array}$ & 85 & 170 & 72.4 & 15.3 & 8.82 & 1.76 & 1.18 & 0.59 & 0 & 0 & 0 & 0 & 0 & 0 & 0 & 0 & 0 & 0 \\
\hline & $\begin{array}{l}\text { Africans } \\
\text { (ASW-1 K) }\end{array}$ & 61 & 122 & 87.7 & 8.20 & 1.64 & 0.82 & 0 & 0 & 0.82 & 0 & 0 & 0 & 0 & 0 & 0 & 0 & 0 & 0.82 \\
\hline Worldwide mean & & 2,171 & 4,342 & 81.0 & 12.2 & 2.14 & 0.57 & 0.65 & 0.16 & 0.68 & 0.99 & 0.14 & 0.07 & 0.09 & 0.15 & 0.07 & 0.03 & 0.07 & 0.01 \\
\hline (range) & & & & $(6.3-100.0)$ & $(0.0-93.8)$ & $(0.0-10.9)$ & $(0.0-7.1)$ & $(0.0-8.0)$ & $(0.0-2.2)$ & $(0.0-9.6)$ & $(0.0-18.2)$ & $(0.0-5.0)$ & $(0.0-2.9)$ & $(0.0-5.6)$ & $(0.0-10.0)$ & $(0.0-2.2)$ & $(0.2-2.2)$ & $(0.0-5.0)$ & $(0.0-0.6)$ \\
\hline
\end{tabular}


are described in reference [25] and of the rest of the alleles in the supplementary (Additional files 1 and 2).

\section{OCT1 activity assays}

The OCT1-mediated uptake was measured essentially as described before [3, 4, 25]. The OCT1-mediated uptake of tropisetron, debrisoquine, O-desmethyltramadol, and monocrotaline was measured using $\mathrm{HPLC}$, of $\mathrm{ASP}^{+}$ using fluorescence spectroscopy and of radiolabelled $\mathrm{MPP}^{+}, \mathrm{TEA}^{+}$, morphine, metformin, and tyramine using scintillation counting. The procedures are described in details in the supplementary (Additional file 1).

\section{Immunofluorescence confocal microscopy and western blot analysis}

The cells were trypsinized with $3.5 \mathrm{~mL}$ TryplLE ${ }^{\mathrm{TM}}$ Express (Life Technologies) for $4 \mathrm{~min}$ at $37{ }^{\circ} \mathrm{C}$ and passed through a $40 \mu \mathrm{m}$ strainer (BD Falcon, Heidelberg, Germany). Two hundred thousand cells were seeded per well of four-well chamber slides (Lab-Tek ${ }^{\mathrm{Tm}}$ II, Nunc, Wiesbaden, Germany), which were pre-coated with poly D-lysine. The next day the cells were washed with phosphate buffered saline (PBS), fixed for 20 min with $4 \%$ formaldehyde, washed twice with PBS, and permeabilized for $15 \mathrm{~min}$ in blocking buffer $(0.5 \%$ fetal calf serum (FCS), $0.1 \%$ bovine serum albumin (BSA), and $0.05 \%$ Triton X-100 in PBS). The cells were incubated with the primary antibodies diluted in blocking buffer. For primary antibodies we used mouse monoclonal antiOCT1 (2C5; Novus Biologicals, Cambridge, UK) diluted 1:400, rabbit monoclonal anti- $\mathrm{Na}^{+} / \mathrm{K}^{+}$ATPase (EP1845Y) diluted 1:200, or rabbit polyclonal anti-Calnexin (ab22595; both obtained from Abcam, Cambridge, UK) diluted 1:1000. After $1 \mathrm{~h}$ incubation at room temperature, the cells were washed three times for 5 min with blocking buffer and incubated with the secondary antibodies for $1 \mathrm{~h}$ in blocking buffer supplemented with $2.5 \mathrm{pg} / \mathrm{mL}$ DAPI (Life Technologies). The incubation was performed at room temperature with samples protected from direct light. As secondary antibodies we used Alexa Fluor 488 goat anti-mouse or Alexa Fluor 546 goat anti-rabbit IgG diluted 1:500 (A-11001 and A11010, respectively; Life Technologies). The cells were washed three times for 5 min with blocking buffer and once with PBS and mounted with Fluoromount-G ${ }^{\mathrm{m}}$ (Southern Biotech; Birmingham, AL, USA). The samples were analyzed on a laser scanning microscope (LSM 710; Carl-Zeiss, Oberkochen, Germany) and the images were processed using ImageJ software v. 1.47 (NIH, Bethesda, MD, USA) with additional contrast adjustment for each channel.

The western blot was performed with total protein extracts of HEK293 cells stably transfected with the different OCT1 variants. The detection was carried out using mouse monoclonal anti-OCT1 (2C5; Novus Biologicals,
Cambridge, UK) and monoclonal anti-GAPDH (6C5; Zytomed, Berlin, Germany) as primary antibodies and horseradish peroxidase-conjugated anti-mouse antibody (BioMol, Hamburg, Germany) as secondary antibodies. Procedure is described in detail in the supplementary.

\section{Computational analyses}

Individual OCT1 haplotypes were inferred using PHASE version 2.1 [26]. Ten independent runs with different seeds $(2,1,536,2,936,3,123,4,957,5,283,6,757,7,992$, $8,633,9,045)$ were performed to exclude seed-biased assignments.

Effects of the non-synonymous variants in OCT1 on protein function were predicted using eight different tools: PROVEAN version1.1.3, SIFT version 1.0.3, PolyPhen-2 version 2.2.2, MutPred version 1.2, Mutation t@sting version 2, SNPs3D, SNAP, and PhD SNP (for details see Additional file 3).

Analysis of molecular variance (AMOVA) was performed on three levels, within population, among populations within each geographic region and among the geographic regions. The calculations were done using Arlequin v3.5.1.2 [27] for the 62 populations in six world regions worldwide. The admix populations from the 1000 Genome Project were excluded from the analysis. Data were entered as DNA sequence data (haplotypic) as the input method for the 16 haplotypes, while we used the allele frequency input method for the phenotypes. Phenotypes were coded as 0,1 , and 2 referring to zero, one, and two active OCT1 alleles, respectively.

The calculations of the average nucleotide diversity $(\pi)$, the estimation of the population mutation parameter $(\theta)$, and the test for neutrality (Tajima's D) were performed using Arlequin v3.5.1.2 [27]. The values for $\pi$ and $\theta$ were normalized to the size of the coding region of the OCT1 gene. All parameters were calculated independently for the six major world regions, and the nine admixed population of the 1000 Genomes Project were excluded from the analysis.

\section{Results}

\section{Global genetic variability in OCT1}

Applying semiconductor-based massively parallel sequencing, we analyzed the global genetic variability in the coding regions of $O C T 1$. We resequenced 2,770 bp of the OCT1 gene, including the complete coding region, the untranslated regions and the intron-exon junctions (Additional file 4). We obtained more than 7.1 million mapped sequencing reads. The average coverage depth was 173 -fold per sample (range 17 to 402) with 977 samples $(91.3 \%)$ having average coverage above 50 -fold. The complete sequencing dataset and individual genotypes were deposited and are freely available in the HGDPCEPH Database [28]. 
We identified a total of 85 variants (a detailed list of all variants is available in Additional file 5). Thereof 44 variants were within the coding region of OCT1 and 29 of them caused amino acid substitutions. We selected 21 amino acid substitution variants for our subsequent analyses (see Fig. 1b for the selection strategy). Included were 14 amino acid substitutions that may affect OCT1 activity (Fig. 2a): nine variants previously known to cause loss of OCT1 activity (Ser14Phe, Arg61Cys, Cys88Arg, Pro117Leu, Ser189Leu, Arg206Cys, Gly401Ser, Met420-deletion, Gly465Arg) [2-5, 7, 10, 11, 17] and five variants predicted to cause loss of OCT1 function (Ser29Leu, Thr245Met, Glu284Lys, Gly414Ala, Ile449Thr). To predict loss of OCT1 function we used eight independent prediction tools. Variants that were predicted to be deleterious by at least five of the eight prediction tools were regarded as potential loss-offunction variants (Additional file 6). We also genotyped two variants, Gln97Lys and Gly220Val, which were not identified in our population by massively parallel sequencing. However, Gln97Lys was previously reported in the literature to strongly affect OCT1 activity [17], while Gly220Val was observed in the 1000 Genomes project and was predicted to be deleterious (Additional file 6). Furthermore, we analyzed five amino acid substitutions (Leu160Phe, Pro341Leu, Arg342His, Met408Val, and Arg488Met) that were known to either not affect or to cause less than $50 \%$ reduction in OCT1 activity $[2,10,11,20,21]$. We included these variants for two reasons. First, we wanted to obtain information about the effects of these variants on the uptake of a much broader spectrum of substrates than previously tested. Second, we aimed to identify common amino acid substitutions that do not affect OCT1 activity as a control in our population genetic analyses.

The methionine 420 deletion (Met420del) was the most commonly observed loss-of-function variant among the 1,079 individuals from 53 populations analyzed (worldwide minor allele frequency of $14.1 \%$ ). The other common loss of function variants were Arg61Cys (3.2\%), Gly465Arg (1 \%), Ser14Phe (0.8 \%), and Gly401Ser (0.7 \%). The variants Ser29Leu, Cys88Arg, and Pro117Leu were observed only in two, eight, and four unrelated individuals, respectively. The variants Ser189Leu, Arg206Cys, Thr245Met, Glu284Lys, and Ile449Thr were observed only in single individuals. The variant Gly220Val was observed in a single individual in the 1000 Genomes sample and was missing in the HGDP-CEPH samples. The variant Gln97Lys was not observed in either sample set.

Among the loss-of-function OCT1 variants, Met420del was the only ubiquitous OCT1 variant that was observed on all continents (Fig. 2b). The Ser14Phe, Arg342His, and Arg488Met variants were frequent in
Africa and occasionally observed in the Middle East and Central Asia, but were completely missing in Europe and America. In contrast, the variants Arg61Cys and Gly465Arg were frequent in Europe, occasionally observed in the Middle East and Central Asia, but completely missing in Africa and America. The rare variants Ser29Leu, Pro117Leu, Arg206Cys, Thr245Met, Glu284Lys, and Ile449Thr were observed only in Asians populations. Among the variants that were not expected to strongly affect OCT1 function, Met408Val was the most frequent. Pro341Leu was the second-most frequent variant. It was observed in all the world regions, but was more common in East Asia.

Comparison with the primate OCT1 sequences showed that, with the exception of serine ${ }_{14}$ and arginine $_{488}$, all the major alleles in humans were also the ancestral alleles (Additional file 7). The primates have phenylalanine at codon 14 and methionine at codon 488 while the major allele in humans is serine ${ }_{14}$ and arginine ${ }_{488}$, respectively.

\section{OCT1 alleles and their functional characteristics}

Using haplotype inferring of the extended HGDP-CEPH and the 1000 Genome Project data we identified 30 haplotypes (Fig. 3a). The haplotypes were grouped to 16 major alleles (designated "1 to "16) and 14 sub-alleles (designated with a capital letter after the major allele number, for example, "1A, "1B, and so on). As suballeles we defined alleles that do not showed more than $50 \%$ reduction or $50 \%$ increase of the activity compared to the corresponding major allele with any of the substrates tested (for details see Fig. 4 and the text below). The non-synonymous variants causing substantial changes in OCT1 activity the variants Cys88Arg, Arg206Cys, and Gly465Arg were not observed alone, but in combination with the Met420del variant (Fig. 3b).

Whereas the allele structure was the same in all populations studied here, the frequencies of the alleles varied substantially among the world regions. The global population frequencies of the major OCT1 alleles are shown in Table 1 and detailed information regarding the allele frequencies of the sub-alleles is given in Additional file 8. Next to the major $O C T 1 * 1$ allele, OCT1*2 was the only evolutionary old major allele observed in all populations. OCT $1 * 2$ was the only loss-of-function allele that was observed in Native Americans. Alleles *3 to *8 were regionspecific: " 3 was observed in Europe, the Middle East and Central Asia, "7 and "8 were observed in Africa, the Middle East, and occasionally in Central Asia. Alleles *9 to "16 were very rare on a global scale (allele frequencies of below $0.5 \%$ ). However, in some specific populations their frequencies may be much higher, for example, the OCT1*12 allele frequency reached $10 \%$ in the population of She Chinese (Table 1). 


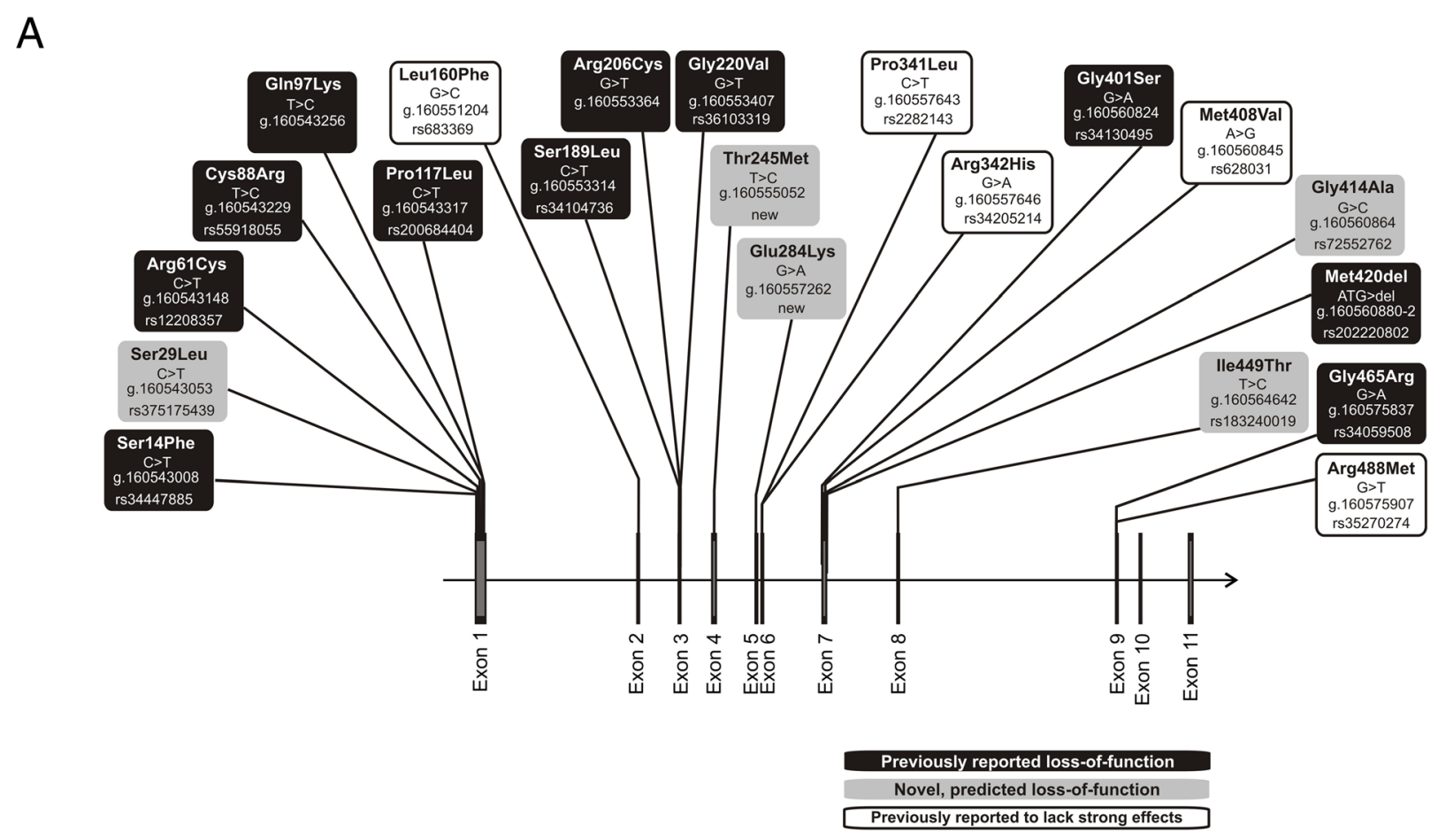

B
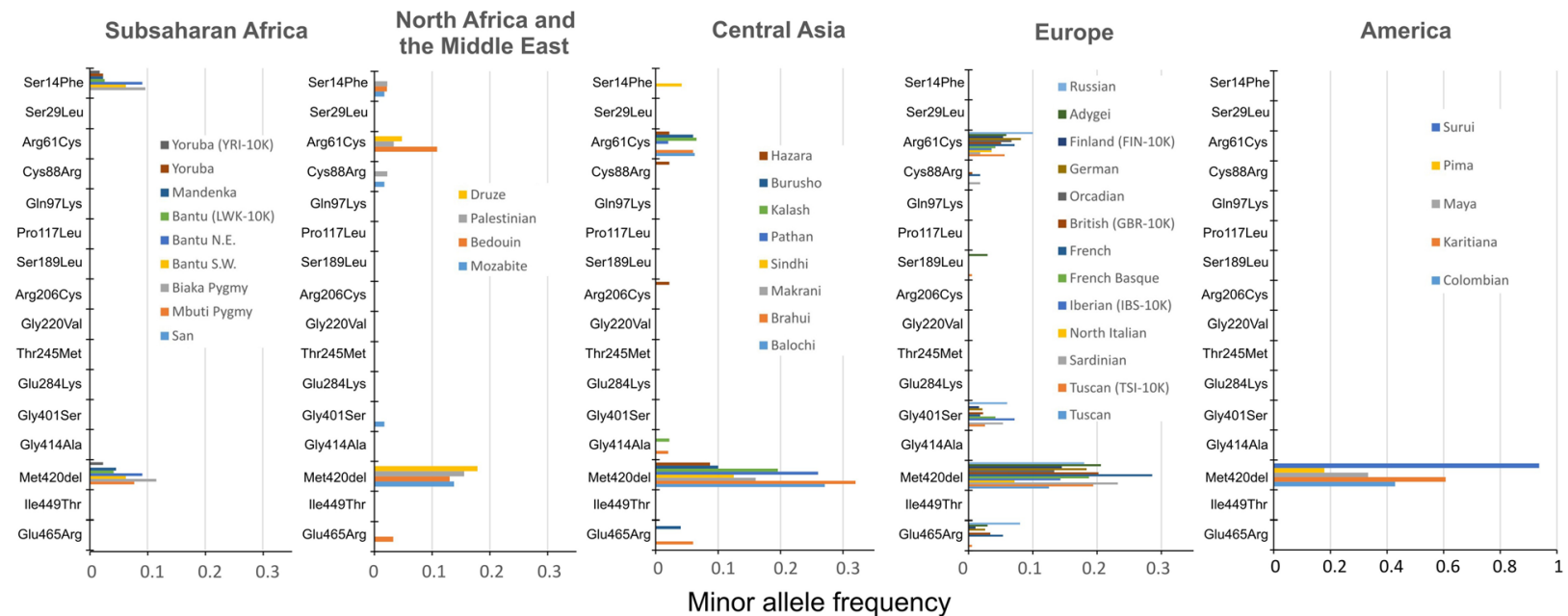

Fig. 2 Non-synonymous OCT1 polymorphisms causing potentially functional amino acid substitutions, and their distribution in different world regions. a The localization of the 21 polymorphisms analyzed in details in this study. Substitutions previously reported in the literature to have strong functional effects on OCT1 activity are shown in black (reported loss of function), substitutions predicted to affect OCT1 activity are shown in gray (predicted loss-of-function), and substitutions previously reported to not affect or to cause less than $50 \%$ reduction of OCT1 activity are shown in white (reported to lack strong effects). The polymorphisms are designated with the amino acid substitutions that they cause and the codon that is affected. Their rs-number in the dbSNP database (if available) and their location on chromosome 6 according to the human genome assembly hg19 are also given. b Minor allele frequencies of the 16 known or predicted loss-of-function OCT1 polymorphisms. Shown are 39 populations from Sub-Saharan Africa, North Africa and the Middle East, Central Asia, Europe, and America. The 39 populations shown include 35 populations from the CEPH human genome diversity panel and five from the 1000 Genome Project [24] (designated with $1 \mathrm{~K}$ ). The populations CEU, ASW, MXL, PUR, and CLM from the 1000 Genomes Project were omitted from these analyses due to their highly admixed structure and/or inability to be allocated to a defined world region 


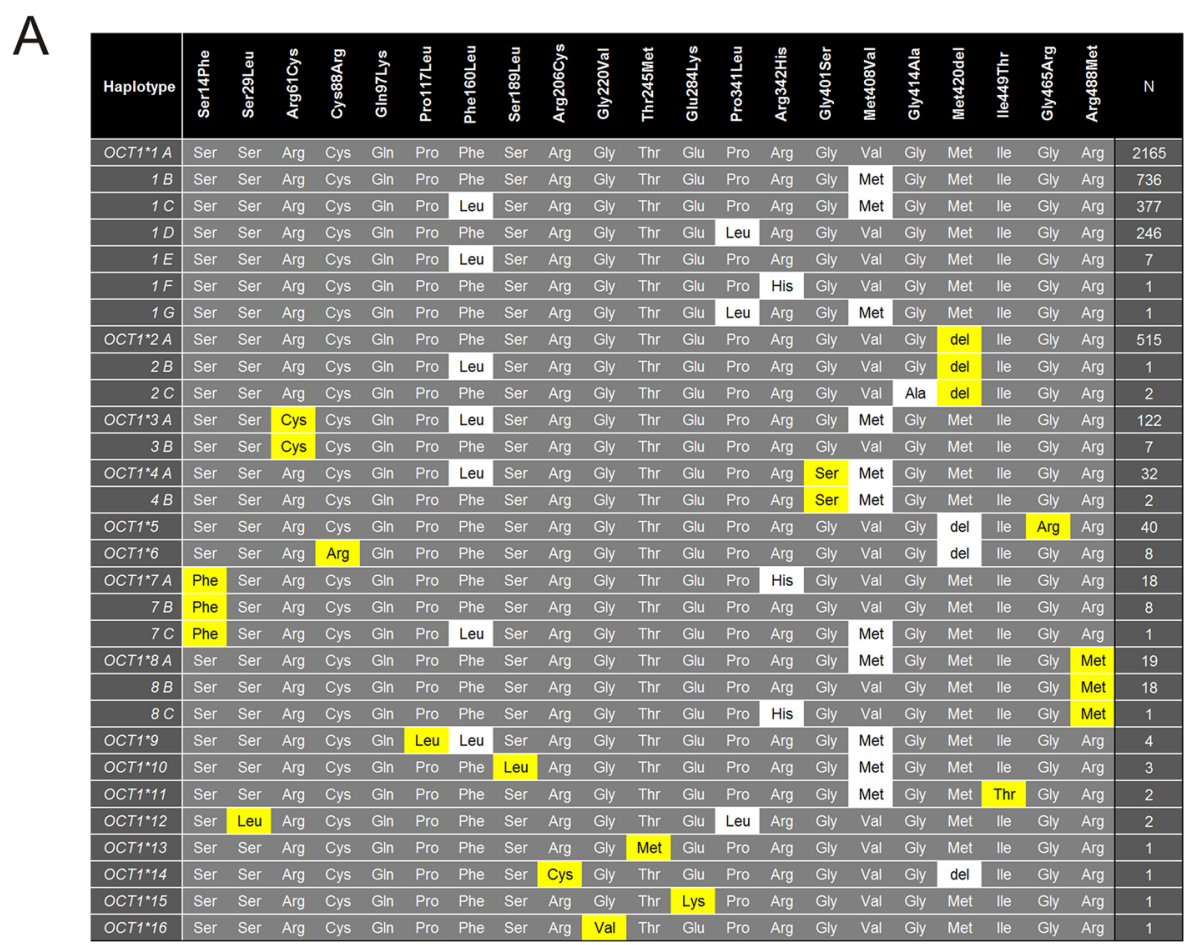

B

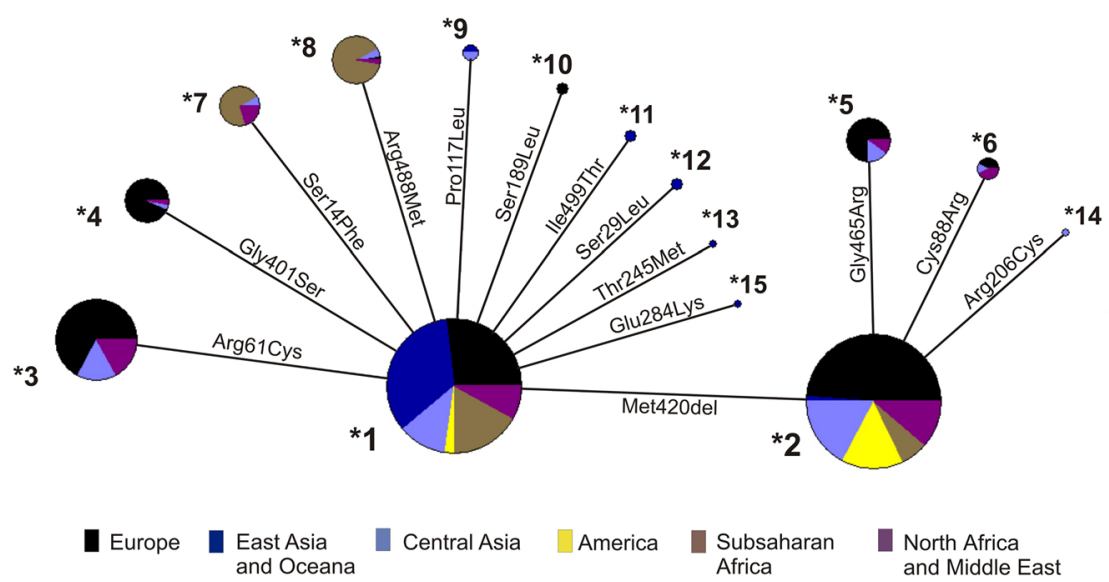

Fig. 3 OCT1 alleles and their global distribution. a Shown are the haplotype combinations constituting the 16 major and 14 sub-alleles of OCT1 observed in the worldwide genetic analyses. As major we defined alleles carrying amino acid substitutions (highlighted in yellow) that cause a more than $50 \%$ decrease or $50 \%$ increase of OCT1 activity in comparison to the reference OCT1*1 allele for at least one substrate tested. The sub-allele of a major allele differs by amino acid substitutions (highlighted in white) that do not affect OCT1 activity. For details about variants affecting or not affecting OCT1 activity see Fig. 4 and the results section in the text. b Cladogram illustrating the relations between the major OCT1 alleles and their worldwide distribution. Different world regions are indicated in colors. Pie size represents the global allele frequency. The amino acid substitutions that distinguish between the alleles are shown. Allele OCT1*16 is missing from the cladogram as it was observed only in admixed population from the 1000 Genomes Project and could not be assigned to any world region. The cladogram was generated using the median joining algorithm of the NETWORK software version 4.6 (Fluxus Technology Ltd.)

Next, we performed functional analyses of 19 of the 21 non-synonymous variants. Two non-synonymous variants, Gln97Lys and Gly220Val, were omitted from the analyses. Gln97Lys was not observed in any of the samples studied here. Gly220Val was observed only in a single individual from an admixed population that could not be used in the global loss of OCT1 function analyses. The 19 variants tested constituted 22 alleles: the 15 major alleles and the seven most common suballeles. The 22 alleles tested represent a total of 4,321 of the 4,342 chromosome 6 copies of the individuals analyzed in this study (Fig. 3a). We characterized the functional activity of the OCT1 alleles in stably transfected HEK293 cells. The transfection resulted in a strong and 


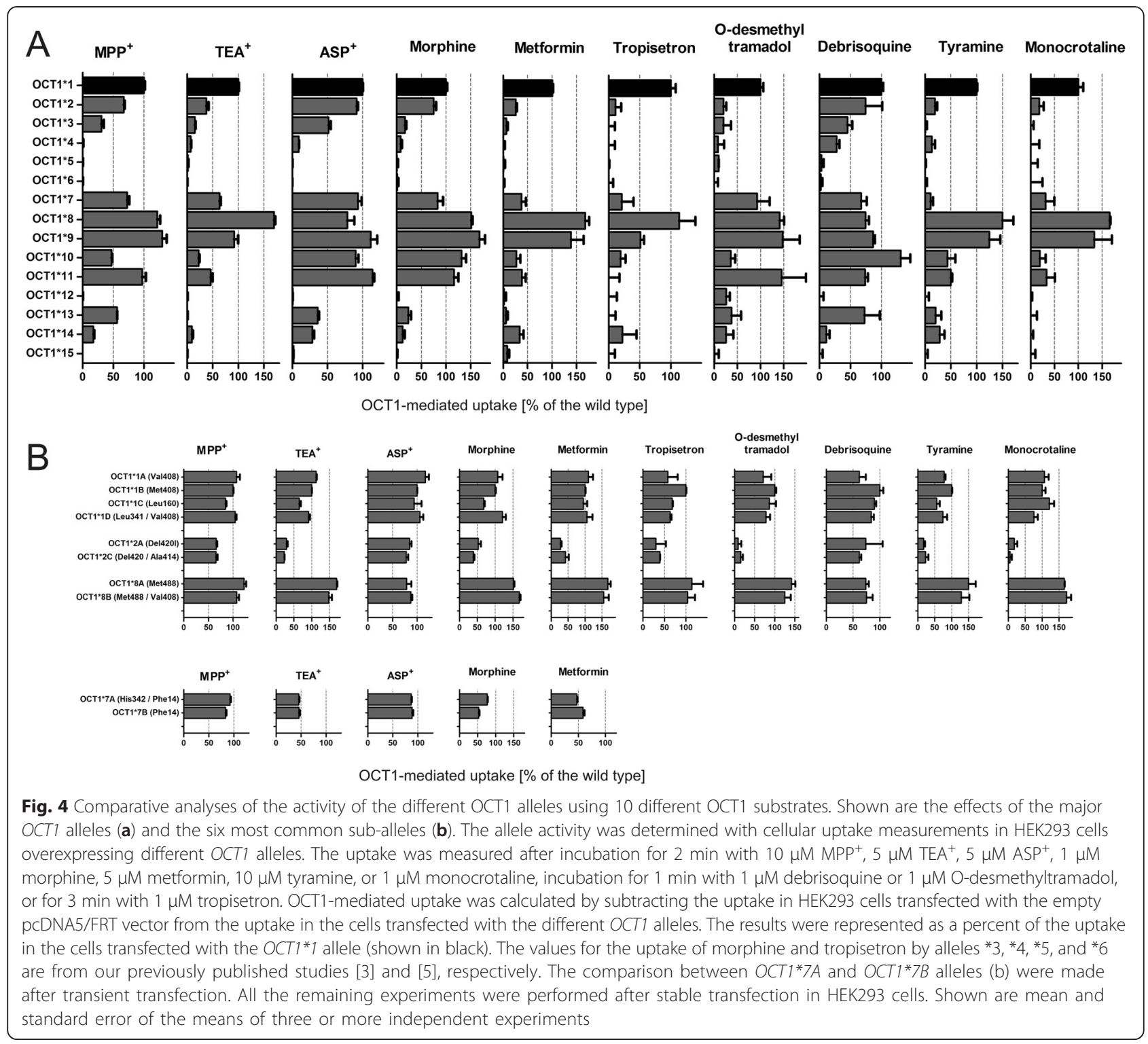

constant overexpression of the mRNA of the different OCT1 alleles (Additional file 2). We measured the ability of the isoforms encoded by the different alleles to transport the model OCT1 substrates 1-methyl-4-phenylpyridinium $\left(\mathrm{MPP}^{+}\right)$, tetraethylammonium $\left(\mathrm{TEA}^{+}\right)$, and 4-(4-(dimethylamino)styrl)-N-methyl-pyridinium $\left(\mathrm{ASP}^{+}\right)$, the drugs debrisoquine, morphine, metformin, tropisetron and $\mathrm{O}$ desmethyltramadol (the active metabolite of tramadol), and the naturally occurring substances tyramine and monocrotaline (Fig. 4a). Tyramine is a biogenic amine suggested to be an OCT1 substrate [29] and was transported by OCT1*1 in our hands with a $\mathrm{K}_{\mathrm{M}}$ of $94.7 \mu \mathrm{M}$ and $\mathrm{V}_{\mathrm{MAX}}$ of $381 \mathrm{pmol} / \mathrm{mg}$ protein $/ \mathrm{min}$ (Additional file 9).

The overexpression of wild-type OCT1 (OCT1*1) resulted in an increase in the uptake of the substances tested by at least 1.9-fold (range 1.9- to 24.1-fold,
Additional file 10). The non-synonymous variants Phe160Leu, Pro341Leu, Arg342His, Met408Val, and Gly414Ala did not cause a more than $50 \%$ decrease or $50 \%$ increase in OCT1 activity with any of the substrates tested (Fig. 4b). Therefore haplotypes carrying these variants were designated as sub-alleles (Fig. 3).

In addition to the sub-alleles, which did not show strong changes to the $O C T 1 * 1$ activity, we identified two gain-of-function OCT1 alleles (the major alleles $O C T 1 * 8$ and $O C T 1 * 9)$ and 11 loss-of-function alleles (the major alleles OCT1*2 to $* 7$ and $O C T 1 * 10$ to $* 15$; Fig. 4a). The allele $O C T 1 * 8$ (characterized by the presence of the Arg488Met substitution) showed a more than $50 \%$ increase in the uptake of $\mathrm{TEA}^{+}$, morphine, metformin, and monocrotaline. The allele OCT1*9 
(Pro117Leu) showed $68 \%$ increase in the uptake of morphine.

The major alleles showing loss of activity could be also separated in three independent groups. The alleles $* 5$ (a combination of Gly465Arg and Met420del), "6 (a combination of Cys88Arg and Met420del), "12 (Ser29Leu), and "15 (Glu284Lys) showed complete loss of OCT1 activity independent of the substrate used (Fig. 4). The alleles *3 (Arg61Cys), *4 (Gly401Ser), and "14 (a combination of Arg206Cys and Met420del) showed strong, but not complete, loss of activity also independent of the substrate. In contrast, the alleles *2 (Met420del), "7 (Ser14Phe), "10 (Ser189Leu), "11 (Ile449Thr), and "13 (Thr245Met) showed substrate-specific loss of activity. The transport activity of alleles "2, "7, "10, and "11 was reduced to $0 \%$ of the OCT1* 1 activity with some of the substrates tested, but was more than $90 \%$ for others (Fig. 4a).

We analyzed the combined effects of the Met420del and Met408Val variants on OCT1 activity in terms of affecting subcellular localization and activity of OCT1. Neither the presence of the Met420 deletion, nor Met408Val affected the correct localization of OCT1 at the plasma membrane (Additional file 11). The presence of Met420del resulted in a substrate-specific loss of OCT1 activity. The Met408Val substitution did not cause differences in the transport of metformin, tyramine, or debrisoquine. However, a small but significant increase of morphine uptake was observed when methionine $_{408}$ was substituted to valine suggesting that when morphine is used as a substrate OCT1*2 allele should be regarded as a reduced activity allele (and not as a complete loss of activity allele as suggested earlier [3]).

We analyzed the ability of model substrates to predict the ability of the alleles that conferred substrate-specific loss of OCT1 activity ("2, "7, "10, "11, and "13) to transport the clinically relevant drugs metformin and morphine (Additional file 12). There was no single model substrate that was able to accurately predict the effect of OCT1 variants on the uptake of both drugs. Whereas morphine uptake correlated better with $\mathrm{ASP}^{+}$uptake, metformin uptake correlated better with $\mathrm{TEA}^{+}$uptake. These results underline the substrate-specific effects of variants $* 2, " 7, * 10, * 11$, and $" 13$ with the consequence that there will not be a single model substrate completely reflecting the effects of these genetic variants on OCT1 activity.

Next we analyzed the subcellular localization of the OCT1 isoforms encoded by the 14 major alleles and the four most common sub-alleles. Most isoforms with substrate-wide loss of function failed to localize in the plasma membrane (Fig. 5a). Co-localization with calnexin suggested retention in the endoplasmic reticulum.
Therefore, lack of correct localization was the major reason for substrate-wide loss of OCT1 activity. The only exception was the allele OCT1*4 (Gly401Ser). OCT1*4 was correctly localized in the plasma membrane, but had between 0 and $28 \%$ of the wild type activity in the substrates tested (Fig. 4a). In contrast, the isoforms with substrate-dependent loss of activity showed normal cellular localization (Additional file 13).

The lack of plasma membrane localization correlated with an altered glycosylation pattern of OCT1 (Fig. 5b). Western blot analyses showed that the variants that were not properly localized in the plasma membrane (OCT1*5, *6, *12, "15, and partially *3 and *14) lacked their PNGaseF-sensitive and EndoH-resistant glycosylation, but kept their EndoH-sensitive glycosylation.

\section{Global variations in loss of OCT1 activity}

Finally and most importantly, we analyzed the frequency of genotypically predicted loss of OCT1 activity worldwide. To this end we categorized the individuals as carriers of two, one or zero completely active OCT1 alleles. We regarded the OCT1 alleles " 1 , "8, and "9 as completely active. The remaining major alleles *2, $3,{ }^{*} 4, * 5$, "6, "7, "10, "11, "12, "13, "14, and "15 we regarded as loss of activity as they show more than $65 \%$ reduction in the OCT1 activity with at least one substrate tested (Fig. 4a).

Between Europeans there were only minor differences in frequency of loss of OCT1 activity (Additional file 14). On average $53 \%$ of the Europeans carried two, $38 \%$ one and $8 \%$ zero active OCT1 alleles. These values were similar to those reported before $[4,10,11]$. A slightly higher frequency of loss of OCT1 activity was observed in Russia and a slightly lower frequency in Northern Italy, but this may be due to the small size of the population samples (25 and 14 individuals, respectively).

Worldwide, we observed strong variations in the frequency of loss of OCT1 activity (Fig. 6). Whereas all individuals from Japan and Oceania carried two completely active OCT1 alleles, the majority of the Surui Indians carried two completely inactive OCT1 alleles. Out of eight Surui Indians analyzed, one (13\%) carried only one and the remaining seven $(87 \%)$ completely lacked any active OCT1 alleles. Similarly to the individuals from Japan, more than $98 \%$ of the Han Chinese, the major population in China, carried two completely active OCT1 alleles and the remaining less than $2 \%$ carried one (Additional file 14). Similar to the Surui, other South American Indians had high frequencies of loss of OCT1 activity, for example, only $7 \%$ of the Karitina Indians carried two completely active OCT1 alleles, $64 \%$ carried one, and $29 \%$ lacked any completely active OCT1 alleles.

Analyses of molecular variance (AMOVA) showed strong variability among the regions of the world at 

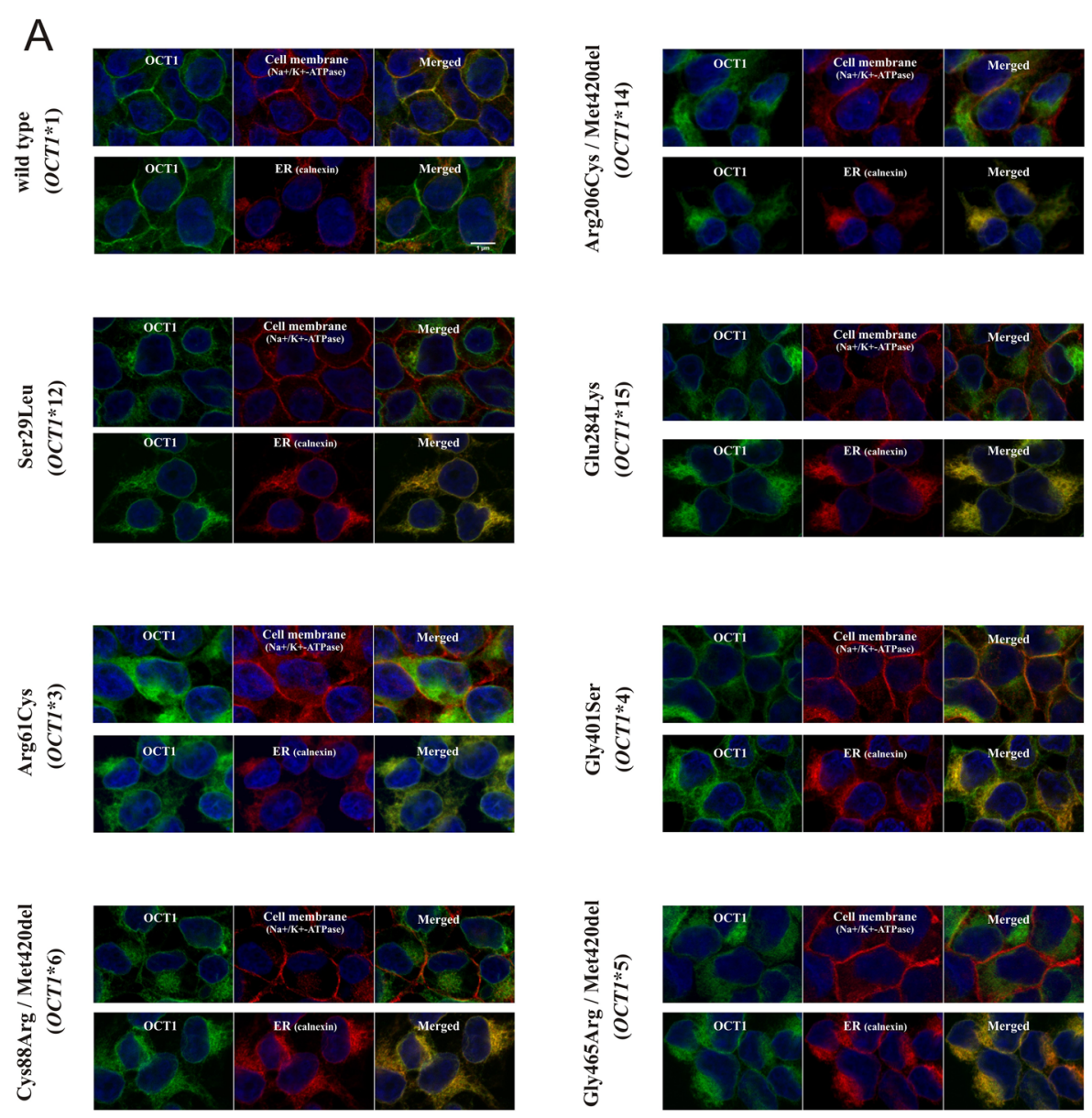

B

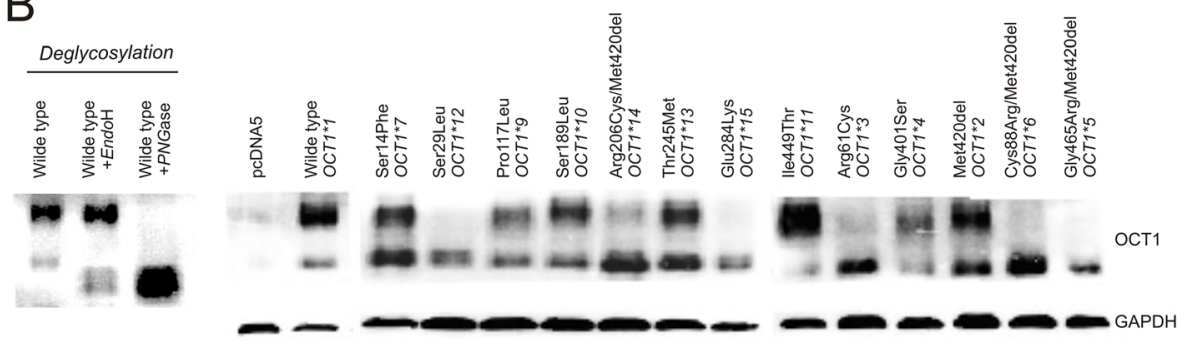

Fig. 5 Subcellular localization and glycosylation patterns of the isoforms encoded by the major OCT1 alleles. a The subcellular localization of the OCT1 isoforms showing that the major cause for substrate-wide loss of OCT1 activity is improper subcellular localization. Shown are the OCT1-overexpressing HEK293 cells stably transfected with alleles causing substrate-wide loss of OCT1 function, and the HEK293 cells expressing the wild type $\left(\mathrm{OCT} 1^{*} 1\right)$ as a reference. The subcellular localization was analyzed with confocal microscopy after immunocytochemical staining for OCT1 (green). The exact OCT1 localization was analyzed using co-staining with $\mathrm{Na}^{+} / \mathrm{K}^{+}$ATPase (red, upper part) as a marker for the plasma membrane and calnexin (red, lower part) as a marker for the endoplasmic reticulum. The immunocytochemical analyses of the sub-alleles and the alleles causing gain or substrate-specific loss of OCT1 function are shown in Additional file 13. b Western blot analyses of total cellular protein illustrating differences between the glycosylation patterns of OCT1 isoforms correctly localized in the plasma membrane and those retained in the endoplasmic reticulum. OCT1 was detected as a double signal of a close to $70 \mathrm{kDa}$ large PNGaseF-sensitive and EndoH-resistant glycosylation form and a $50 \mathrm{kDa}$ EndoH-sensitive glycosylation form. Glyceraldehyde-3-phosphate dehydrogenase (GAPDH) was used as a loading control

haplotype level and even stronger at phenotypic level (10.5\% and $18.7 \%$, respectively; Table 2$)$. Within the different geographic regions the highest variability among the populations was observed by the Native Americans
(36.3\% at phenotypic level) compared to only $1.3 \%$ by the European populations.

Pairwise $\mathrm{F}_{\mathrm{ST}}$ values analyses showed strong divergence in the loss of OCT1 activity between the America and 


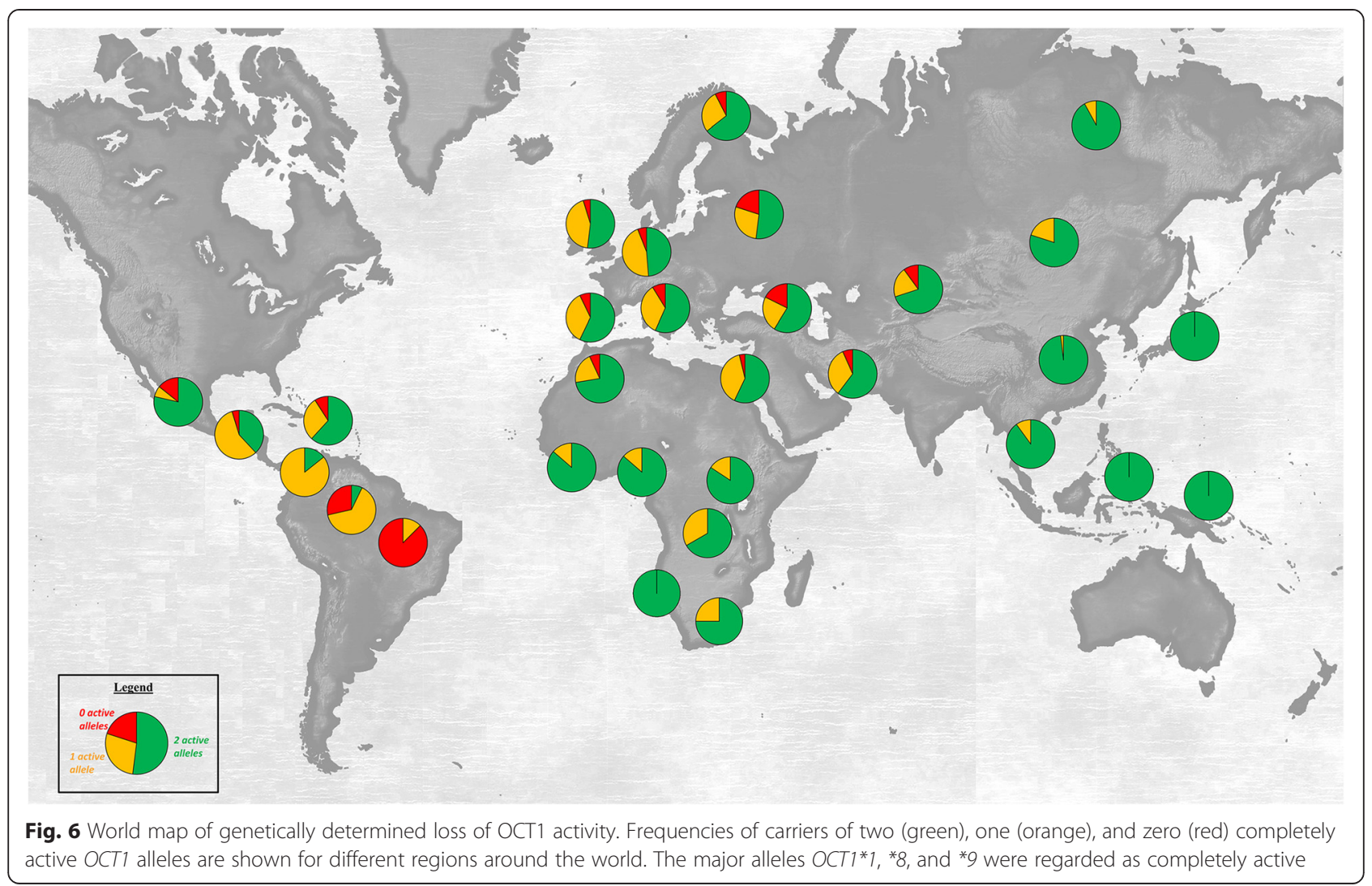

the East Asia populations $\left(\mathrm{F}_{\mathrm{ST}}\right.$ of 0.76 , Fig. $\left.7 \mathrm{a}\right)$. Similar results were obtained by analyzing the OCT1 genetic variability at the genetic level without regarding the functional effects of the haplotypes $\left(\mathrm{F}_{\mathrm{ST}}\right.$ of 0.72 , Additional file 15). There was only limited positive correlation between the frequency of loss of OCT1 activity and the geographic distance to Addis Ababa, which may be regarded as a starting migration point of the modern humans according to the Out-of-Africa model $(\mathrm{r}=0.28, P<0.05$, Fig. $7 \mathrm{~b})$. However, the correlation was much stronger when the populations from East Asia and Oceania were excluded from the analyses $\left(r^{2}=0.640\right.$, $P<10^{-4}$, Fig. 7c).

In addition, we took advantage of the available genotypes of the HGDP-CEPH individuals for another polymorphic drug transporter OATP1B1 [30] and the metabolizing enzyme CYP2D6 [31] and analyzed the correlation between loss of OCT1 activity and loss of OATP1B1 or CYP2D6 activity. We regarded the presence of the c.521C (Ala174) allele as a loss of OATP1B1

Table 2 Global analysis of molecular variance in OCT1 gene

\begin{tabular}{|c|c|c|c|c|c|c|c|c|}
\hline & \multirow{2}{*}{$\begin{array}{l}\text { Number of } \\
\text { regions }\end{array}$} & \multirow{2}{*}{$\begin{array}{l}\text { Number of } \\
\text { populations }\end{array}$} & \multicolumn{3}{|l|}{ Haplotypes } & \multicolumn{3}{|l|}{ Phenotypes } \\
\hline & & & $\begin{array}{l}\text { Within } \\
\text { populations }\end{array}$ & $\begin{array}{l}\text { Among populations } \\
\text { within regions }\end{array}$ & $\begin{array}{l}\text { Among } \\
\text { regions }\end{array}$ & $\begin{array}{l}\text { Within } \\
\text { populations }\end{array}$ & $\begin{array}{l}\text { Among populations } \\
\text { within regions }\end{array}$ & $\begin{array}{l}\text { Among } \\
\text { regions }\end{array}$ \\
\hline World & 1 & 62 & 89.2 & 10.8 & & 79.9 & 20.1 & \\
\hline World & 6 & 62 & 87.4 & 2.1 & 10.5 & 76.9 & 4.4 & 18.7 \\
\hline Sub-Saharan Africa & 1 & 9 & 97.6 & 2.4 & & 91.7 & 8.3 & \\
\hline $\begin{array}{l}\text { North Africa and } \\
\text { Middle East }\end{array}$ & 1 & 4 & 99.9 & 0.1 & & 99.5 & 0.5 & \\
\hline Europe & 1 & 13 & 100.0 & 0.0 & & 98.7 & 1.3 & \\
\hline Central Asia & 1 & 10 & 96.2 & 3.8 & & 91.5 & 8.5 & \\
\hline East Asia and Oceania & 1 & 21 & 94.9 & 5.1 & & 89.5 & 10.5 & \\
\hline America & 1 & 5 & 75.6 & 24.4 & & 63.7 & 36.3 & \\
\hline
\end{tabular}




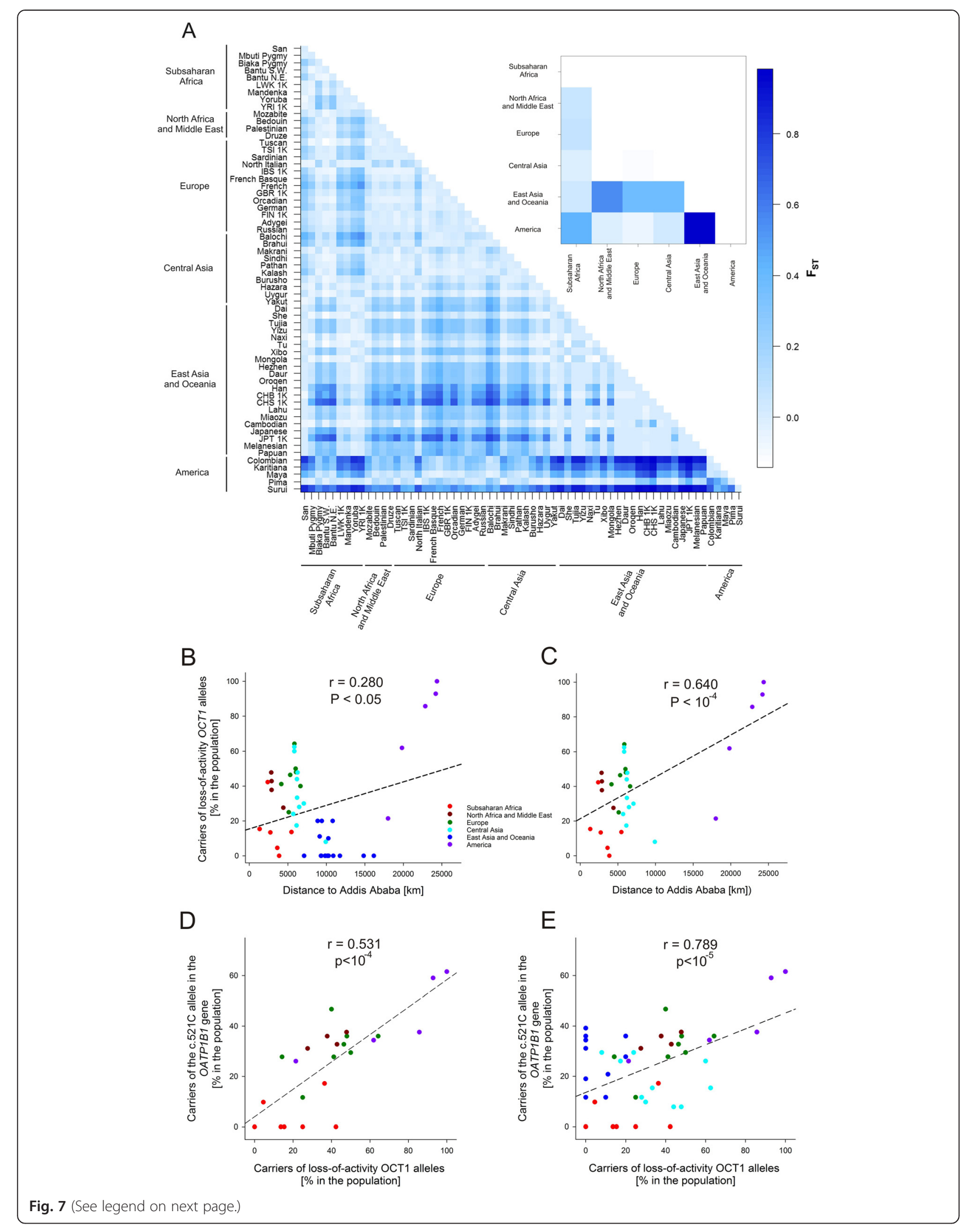


(See figure on previous page.)

Fig. 7 Analyses of the variations in the frequencies of loss of OCT1 activity. a Pairwise comparison of the divergences between the populations and between the world regions (the upper panel). The divergences between the populations were shown in terms of pairwise fixation indexes $\left(F_{S T}\right)$. The deep blue color corresponds to high divergence between the populations / regions (high $\mathrm{F}_{\mathrm{ST}}$ values). We analyzed 62 populations from the 1000 Genomes Project (designated with $1 \mathrm{~K}$ ) and HGDP-CEPH project (the rest). The populations were stratified in world regions as shown in Table 1. The populations CEU, ASW, MXL, PUR, and CLM from the 1000 Genomes Project were omitted from these analyses due to their highly admixed structure and/or inability to be allocated to a defined world region. $\mathbf{b}$ and c Correlation analyses between the loss of OCT1 and geographic distance to Addis Ababa. Shown are all HGDP-CEPH populations (b) and populations outside East Asia and Oceania (c). The distances to Addis Ababa are according to the previously published ones [37]. Shown are Pearson's correlation coefficients. The number of populations analyzed were 50 in (b) and 32 in (c). $\mathbf{d}$ and e Correlation analyses between the loss of OCT1 and of OATP1B1 activity in HGDP-CEPH populations. Shown are all populations (d) and populations outside Asia and Oceania (e). Loss of OATP1B1 activity is represented as the presence of the C-allele of the C. $521 \mathrm{~T}>\mathrm{C}$ polymorphism. The carrier of the C-allele were estimated from the population data of Pasanen et al. [30] assuming Hardy-Weinberg distribution of the genotypes within each population. Shown are Pearson's correlation coefficients. The number of populations analyzed were 52 and 24 in (d) (e), respectively

activity. The c.521C allele was reported both in vitro and in vivo to be the common polymorphism that most strongly affects OATP1B1 activity [32-34]. The frequency of loss of OCT1 activity correlated with the frequency of loss of OATP1B1 activity $\left(\mathrm{r}=0.531, P<10^{-4}\right.$, Fig. 7d). The correlation was much stronger when the Asian and Oceanian populations were excluded from the analyses $\left(\mathrm{r}^{2}=0.789, P<10^{-5}\right.$, Fig. $\left.7 \mathrm{e}\right)$. We regarded the presence of any of the nonfunctional CYP2D6 alleles, CYP2D6 *3, *4, *5, or *6, as a loss of CYP2D6 activity. No correlation was observed between the loss of OCT1 activity and loss of CYP2D6 activity (data not shown).

Next we performed classical population genetic analyses to test for neutrality of the observed loss of OCT1 function. We analyzed the region-specific variation in nucleotide diversities $(\pi)$ of the amino acid substitutions in OCT1 and compared them with the expected average heterozygosity (also known as population mutation parameter $\theta$; Table 3 ). The observed nucleotide diversity in the different world regions was on average $5.88 \times 10^{-4}$, which was very close to the previously reported value $\left(5.11 \times 10^{-4},[16,35]\right)$. The nucleotide diversity varied between $3.6 \times 10^{-4}$ and $7.9 \times 10^{-4}$ among the world regions (Table 3).
We took advantage of the detailed functional analyses that we performed in this study and analyzed separately the nucleotide diversity for the amino acid substitutions causing loss of OCT1 function $\left(\pi_{\mathrm{LOF}}\right)$ and the ones not causing loss of function ( $\left.\pi_{\text {non-LOF }}\right)$. We observed strong, more than 10-fold variations in $\pi_{\mathrm{LOF}}$ among the different world regions (Table 3 ). By far the lowest $\pi_{\text {LOF }}$ was observed in East Asia and Oceania $\left(0.16 \times 10^{-4}\right)$. In addition, the ratio of $\pi_{\mathrm{LOF}}$ to $\pi_{\text {non-LOF }}$ was extremely low for East Asia and Oceania (0.03 compared to an average of 0.89 for the whole world regions, Table 3). Furthermore, the observed loss-of-function nucleotide diversity in East Asia and Oceania was significantly lower than the expected value $\left(\theta\right.$ of $4.8 \times 10^{-4}$; Tajima's D of -1.64 , $P<0.01)$. This indicates the presence of purifying selection for loss-of-function OCT1 variants in this region. In contrast, the ratio of $\pi_{\text {LOF }}$ to $\pi_{\text {non-LOF }}$ variants in Native Americans was very high, but the difference between the observed and expected loss-of-function nucleotide diversity was not significant (Table 3 ).

\section{Discussion}

The comprehensive population and functional genetic analyses performed in this study revealed strong

Table 3 Population genetic statistics of the OCT1 gene for the different world regions

\begin{tabular}{|c|c|c|c|c|c|c|c|c|c|c|}
\hline \multirow[t]{2}{*}{ World region } & \multicolumn{4}{|c|}{$\pi\left(\times 10^{-4}\right)$} & \multicolumn{3}{|c|}{$\theta\left(\times 10^{-4}\right)$} & \multicolumn{3}{|c|}{ Tajima's D } \\
\hline & All & LOF & Non-LOF & $\pi_{\text {LOF }} / \pi_{\text {non-LOF }}$ & All & LOF & Non-LOF & All & LOF & Non-LOF \\
\hline Sub-Saharan Africa & 4.91 & 0.85 & 4.06 & 0.21 & 5.19 & 1.73 & 3.46 & -0.096 & -0.601 & 0.271 \\
\hline North Africa and Middle East & 5.73 & 2.59 & 3.15 & 0.82 & 9.45 & 5.67 & 3.78 & -0.869 & -1.040 & -0.280 \\
\hline Europe & 7.93 & 3.06 & 4.87 & 0.63 & 8.52 & 4.65 & 3.87 & -0.140 & -0.568 & 0.400 \\
\hline Central Asia & 7.60 & 2.36 & 5.24 & 0.45 & 11.73 & 5.42 & 6.32 & -0.803 & -1.042 & -0.330 \\
\hline East Asia and Oceania & 5.48 & 0.16 & 5.32 & 0.03 & 7.99 & 4.79 & 3.20 & -0.621 & $-1.638^{*}$ & 0.976 \\
\hline America & 3.63 & 2.76 & 0.87 & 3.18 & 4.25 & 1.06 & 3.18 & -0.268 & 1.675 & -1.204 \\
\hline
\end{tabular}

LOF, loss-of-function. Included are all variants causing a more than $50 \%$ reduction in OCT1 activity for at least one substrate tested, that is, Ser14Phe, Ser29Leu, Arg61Cys, Cys88Arg, Ser189Leu, Arg206Cys, Thr245Met, Glu284Lys, Gly401Ser, Met420del, lle449Thr, and Gly465Arg; non-LOF, non-loss-of-function. Included are all variants that did not affect or caused an increase in OCT1 activity, that is, Pro117Leu, Phe160Leu, Pro341Leu, Arg342His, Met408Val, Gly414Ala, and Arg488Met ${ }^{*} P<0.01$ 
variability in the frequency of genetically-determined loss of OCT1 activity among populations around the world. Whereas in East Asia lack of OCT1 activity was very rare, lack of OCT1 activity was very common in some native South American populations, who were mostly carriers of the $O C T 1 * 2$ allele. Depending on the substrate, more than $80 \%$ of the Surui Indians completely lack OCT1 activity and the remaining less than $20 \%$ have only one active OCT1 allele. The genetic variability of OCT1 among different world regions was much higher compared to other genes involved in the detoxification of xenobiotics and known for their high genetic variability like CYP2D6. Whereas a previously published analysis of genetic variability of CYP2D6 in the HGDP-CEPH panel found only $6.5 \%$ phenotypic variability among world regions [31], here we observed $18.7 \%$ variability for OCT1 (Table 2).

The observed strong population-specific loss of OCT1 activity may reflect in population-specific differences of pharmacokinetics and therapeutic efficacy of some commonly used drugs. Thus far, most clinical studies on OCT1 polymorphisms have been performed in Caucasians. Using the data from this study, the results may be extrapolated to other populations worldwide. Numerous drugs including metformin, morphine, tramadol, and tropisetron have been suggested to depend on OCT1 transport to be metabolized or to exert their pharmacological effects [2-5]. Furthermore, genetically determined loss of OCT1 activity was found to correlate with variations in the pharmacokinetics and the efficacy of these drugs $[2-5,12]$. Based on the results of this study it should be expected that populations from East Asia and Africa will have generally lower and less inter-individually variable plasma concentrations of these drugs. Indeed, Sadhasivam et al. reported lower plasma concentrations of morphine in the children of African Americans, compared to white Americans [36] and suggested that this may be due to the more common loss of OCT1 activity in Caucasians compared with Africans [12]. This hypothesis is supported by our data. Our analyses showed that in Sub-Saharan Africa only $5 \%$ of the population carry alleles causing a reduction in OCT1-mediated uptake of morphine, in contrast to $25 \%$ in Europe (Table 1 and Fig. 4a).

The highly variable frequency of loss-of-function OCT1 polymorphisms suggests the existence of selection pressure for losing (or maintaining) OCT1 activity in some world regions. The strongest divergence in the frequency of losing OCT1 activity was observed between the populations in America and East Asia (Fig. 7a). Native Americans and East Asians, however, shared a recent common ancestor and have low genetic divergence on a genome-wide scale [37]. This suggests that a selection pressure exists either for maintaining OCT1 activity in East Asia or losing OCT1 activity in America.

Several population genetic analyses pointed to a pressure for maintaining OCT1 activity in East Asia and Oceania. First, and most important, the observed loss-of-function variability in East Asia and Oceania was significantly lower than the expected one (Tajima's $\mathrm{D}=-1.64, P<0.01$; Table 3 ). In addition, the ratio of the loss-of-function and the non-loss-of-function amino acid substitutions was more than 10-fold lower in East Asia and Oceania than in the other world regions. Second, we observed a correlation of moderate significance between loss of OCT1 activity and population migration distance (Fig. 7b). This correlation was strongly improved when East Asian populations were excluded from the analyses (Fig. 7c). In addition, significant correlation was observed between the frequencies of loss of activity of OCT1 and OATP1B1, another genetically variable drug transporter expressed in the liver (Fig. 7d) [30]. This correlation was again substantially improved when East Asian and Oceania populations were excluded from the analyses (Fig. 7e). Taken together these data suggest that a selection pressure for maintaining OCT1 activity in East Asia may be the major cause for the strong divergence in OCT1 activity between East Asia and America. Finding the cause of this pressure may shed a light on the important question about the physiological role of OCT1 in humans.

In other polymorphic genes responsible for drug metabolism and disposition, toxins have been suggested as an important source of selection pressure [38]. In the case of OCT1 selective pressure may come from foodderived organic cations or hydrophilic weak organic bases that are toxic if not rapidly detoxified in the liver. Although the truly responsible component remains unknown, we may illustrate the idea on the examples of tyramine and monocrotaline.

Tyramine is a biogenic amine that is produced by incomplete fermentation from bacteria like Carnobacterium sp. and Lactobacillus sp. [39]. Substantial amounts of tyramine and other biogenic amines are found in numerous types of bacterially fermented foods (cheese, some meat products, and red wine) [40]. Tyramine is detoxified by monoamine oxidases (MAO), which are strongly expressed in the human liver [41]. Failure to detoxify tyramine can result in increased blood pressure, migraine and nausea. Tyramine is a substrate of the human OCT1 ([29] and this work) and the alleles conferring loss of OCT1 activity are not able to transport tyramine (Fig. 4a). Therefore carriers of one and especially of zero active OCT1 alleles may experience increased plasma concentrations and suffer from adverse effects of tyramine. However, tyramine would execute a 
selective pressure for keeping OCT1 and cannot explain the high frequencies of loss of OCT1 activity.

A selection pressure for the loss of OCT1 activity may exist for example if loss of OCT1 activity protects the liver from toxic organic cations. An interesting example is the case of monocrotaline, a pyrrolizidine alkaloid which derives from the Crotalaria genus of flowering plants. Monocrotaline may be ingested as a result of contamination of food or beverages [42]. It undergoes hepatic biotransformation into severely hepatotoxic products [43], in an OCT1-dependent manner [9]. However, common OCT1 polymorphisms result in lack of monocrotaline transport (Fig. 4a) and would thus protect the host against this toxicity. Although the global distribution of Crotalaria sp. that produce these types of toxins [44] does not overlap with the distribution of the loss of OCT1 activity, this substrate is proof of principle that lack of OCT1 activity could protect against ingested toxins. Furthermore, several other hepatotoxic alkaloids were recently reported to be OCT1 substrates $[45,46]$. Alternatively, Oct1 deficiency in mice was reported to protect from hepatic steatosis [1] and therefore may improve fitness. However, it remains open to what extent the same effects are present in humans and it remains elusive why Native Americans would be at a higher risk of hepatic steatosis then populations in other word regions.

Alternatively, the variable frequencies of loss of OCT1 activity may result from genetic drift processes like founder effects and populations bottlenecks. This may be especially true for the high frequency of loss of OCT1 activity in Surui Indians. First, the South American Indians have traveled the longest migration distance among the Homo sapiens populations and experienced multiple founder effects [47]. This explanation is supported by the observed gradual increase in the frequency of loss of OCT1 activity from North to South America (Fig. 6). Second, Surui Indians have experienced a population bottleneck about 45 years ago when more than two-thirds of the Surui population died due to diseases introduced by the first settlers [48]. This can also explain the higher levels of the observed vs. expected nucleotide diversity in Native Americans (Tajima's $\mathrm{D}=1.67$; Table 3).

The average non-synonymous heterozygosity in OCT1 observed in this study (Table 3) was comparable with previous reports $[16,35]$. In the context of the other organic cation transporters of the SLC22 family, the average non-synonymous heterozygosity of OCT1 is comparable with that of OCTN1 and substantially higher than those of OCT2, OCT3, and OCTN2 [16, 35]. In the context of the broader group of pharmacologicallyrelevant membrane transporters, the average nonsynonymous heterozygosity of OCT1 is lower than the values for VMAT1, CNT1, and PEPT2, but is substantially higher than the values of the dopamine and serotonin reuptake transporters $D A T$ and $S E R T$ [16]. However, simple comparisons between synonymous and non-synonymous variability have the limitation that the non-synonymous variants are automatically assumed to affect protein function. Here, after performing very broad substrate analyses, we found that five of the non-synonymous variants ( $26 \%$ of the functionally tested variants) do not substantially affect OCT1 function. These variants were also among the most frequent ones, with substantial contribution to the average heterozygosity. Of the remaining variants, $12(63 \%)$ caused a decrease and another two caused an increase in OCT1 function. Therefore, a broader substrate-wide functional analysis of the nonsynonymous polymorphisms, similar to the analyses presented here for OCT1, may be more useful for addressing the differences between functional and non-functional variability in order to get more precise information on neutrality.

One limitation of our study is the small number of individuals analyzed in some populations. For example, the observed extremely high percentage of loss of OCT1 activity in Surui Indians is based on the analysis of only eight individuals. However, even with this small number of individuals, the probability that this observation has been made by chance and that the frequency of loss of OCT1 activity in Surui Indians is similar to the frequency in Europe (the world region with the highest frequency of loss of activity besides America) or in Brahui individuals (the population with the highest frequency of loss of activity outside of America) is $6.3 \times 10^{-8}$ or $4.8 \times 10^{-6}$, respectively. Still, with respect to the particular case of the extremely high frequency of loss of OCT1 activity in Surui Indians, we regard our data as preliminary results that require validation.

Another important observation in our study is that the major reason for substrate-wide loss of OCT1 activity is improper membrane localization. With one exception (Gly401Ser), all polymorphisms that caused complete (Ser29Leu, Cys88Arg, Glu284Lys, Gly465Arg) or very strong (Arg61Cys and Arg206Cys) substrate-wide loss of OCT1 activity also cause retention of the protein in the endoplasmic reticulum. This leads to lack or a strong reduction of protein expression at the plasma membrane (Fig. 5). Several cysteine residues within the large extracellular loop between transmembrane helices one and two are involved in building intra-molecular disulfide bonds essential for the oligomerization and for the targeting of OCT1 to the plasma membrane [49]. Indeed two polymorphisms causing improper membrane localization, Cys88Arg and Arg61Cys, are located in the large extracellular loop (Fig. 8a). Cys88Arg destroys a cysteine residue known to build disulfide bonds [49] and 


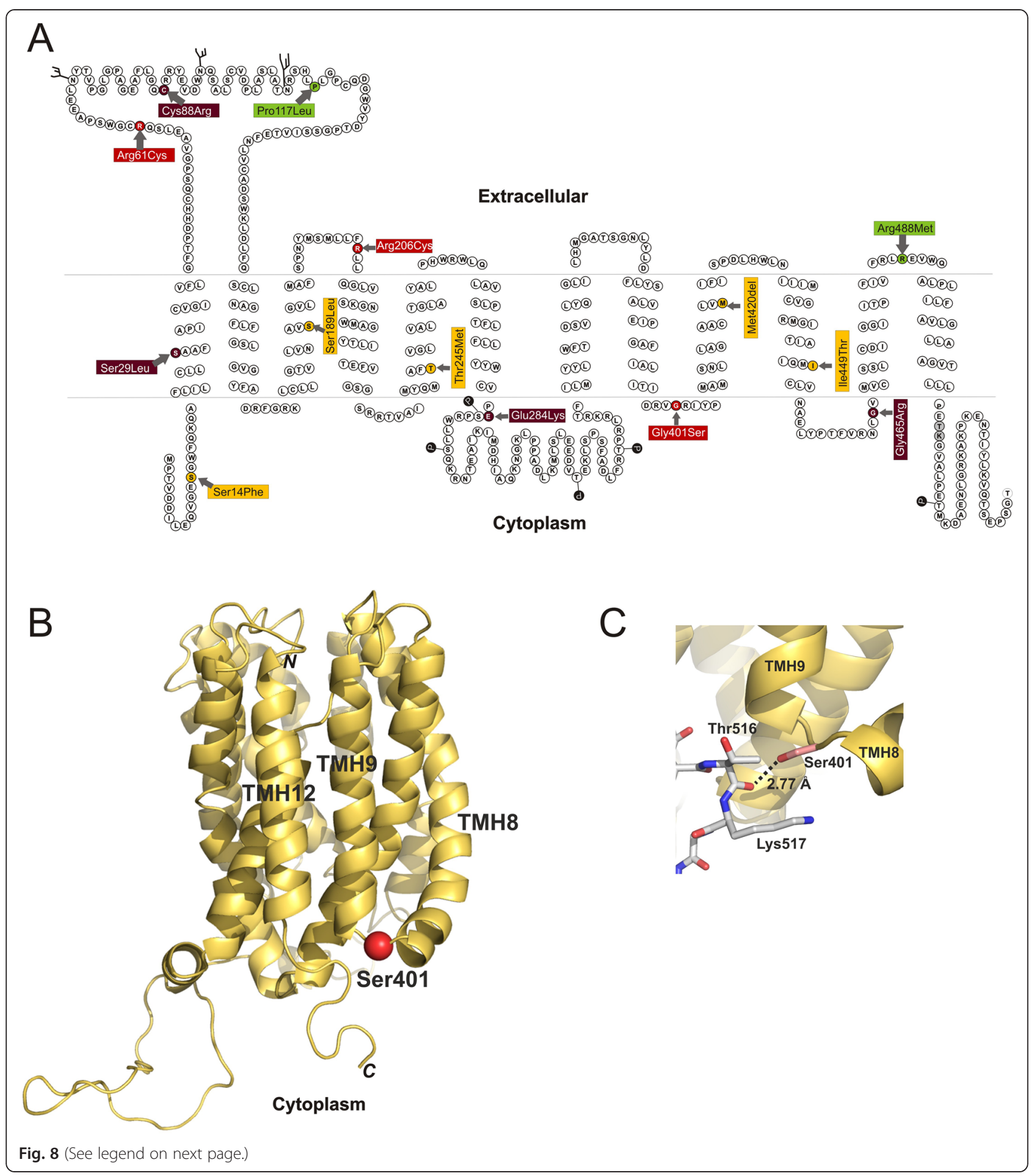


(See figure on previous page.)

Fig. 8 Localization of the functional amino acid substitutions within the OCT1 protein. a Schematic representation of the secondary structure of OCT1 with the positions of the amino acid substitutions causing strong changes in OCT1 activity indicated. Substitutions leading to substrate-specific loss of activity are shown in orange, substitutions leading to partial, but substrate-overarching, loss of activity in red, substitutions leading to complete substrate-overarching loss of activity in dark red, and substitutions leading to gain of function are shown in green. The amino acids threonine ${ }_{516}$ and lysine ${ }_{517}$, which are assumed to be involved in interaction with serine 401 (see below), are shown in gray. Potential phosphorylation $(P)$ and glycosylation $(\psi)$ sites are indicated as they were predicted by Zhang et al. [6]. b A 3D model of the OCT1 protein with the position of the Gly401Ser polymorphism highlighted. Represented is a homology model of the inward-facing conformation of OCT1 (Model-ID Q9NQD4, ModBase [51]) which was visualized using PyMol software version 1.3 (Schrödinger, LLC, München, Germany). The model is based on homology with lactose permease LacY of Escherichia coli (PDB number 1pv6). Gly401Ser substitution is shown as a red sphere. Transmembrane helices TMH8, TMH9 and TMH12, the N terminus and C terminus are indicated. $\mathbf{c}$ A detailed representation of the region of the Gly401Ser substitution indicating a potential hydrogen bond between the hydroxyl oxygen of serine 401 and the carbonyl oxygen

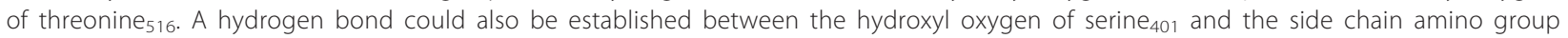
of lysine 517 assuming other rotational conformations for that side chains are allowed. The position of serine ${ }_{401}$, threonine ${ }_{516}$ and lysine 517 are shown in details. Oxygen atoms are depicted in red and nitrogen atoms in blue

Arg61Cys generates an alternative cysteine residue in direct proximity to an already existing one.

Not all polymorphisms that affected the membrane localization are located in the large extracellular loop. Arg206Cys is located in the second extracellular loop between transmembrane helices 3 and 4 (Fig. 8a), but it also generates an extracellular cysteine (or facing the endoplasmic reticulum during the protein synthesis). This cysteine $_{206}$ may interact with extracellular parts of OCT1 or other molecules and act as an 'anchor' preventing proper folding or translocation to the plasma membrane. The other polymorphisms preventing the OCT1 localization in the cell membrane are located either in the first transmembrane domain (Ser29Leu) or in the intracellular loops (Glu284Lys and Gly465Arg). The Glu284Lys and Gly465Arg are changes in amino acids that are highly conserved between species (orthologs) and also between the members of the SLC22 family (paralogs, see Additional file 7). The members of the SLC22 family sharing this conservation recognize completely different substrates than OCT1 does. Therefore, it could be speculated that these highly conserved substrate-unspecific parts of the protein may have important structural functions and polymorphism affecting them lead to improper structure of the protein and its retention in the endoplasmic reticulum.

Gly401Ser apparently causes general impairment of the transport process without affecting the membrane localization of OCT1. Gly401Ser is located in the intracellular loop between transmembrane helices 8 and 9 (Fig. 8a). Molecular modeling suggests that this loop is in close proximity to the $\mathrm{C}$-terminal end of transmembrane helix 12 and to the intracellular C-terminal tail of the protein thereafter (Fig. $8 \mathrm{~b}$ ). When the structurally flexible glycine ${ }_{401}$ is substituted with serine, a possible interaction may occur between the hydroxyl group of serine $_{401}$ and the protein backbone between threonine ${ }_{516}$ and lysine ${ }_{517}$ (Fig. 8c). Accounting for the experimentally observed substrate-wide loss of OCT1 activity, we may speculate that this interaction leads to impairment of a general process like the protein plasticity essential for the transmembrane translocation of the substrate.

A substantial number of the non-synonymous polymorphisms studied showed substrate-specific loss of OCT1 activity. Five out of all 19 non-synonymous polymorphisms tested (26\%) and out of the 12 polymorphisms causing loss of OCT1 activity (42\%) showed substrate-specific effects. This number is in line with the analyses of Urban et al. who reported that $17 \%$ of the non-synonymous variants in drug transporters including OCT1 show substrate-specific effects [35]. However, the number given by Urban et al. may be an underestimate, as it is based on the comparison of the effect of the polymorphism on only two substrates. Most polymorphisms causing substrate-specific loss of OCT1 activity are located in the transmembrane helices that are known to be involved in the formation of the binding cleft (Fig. 8) [50]. Although none of them affect amino acids known to be involved in the substrate binding [50], they do show up to $100 \%$ reduction in the transport activity with some substrates (Fig. 4). Substrate-specific effects of the Met420del polymorphism $(O C T 1 * 2)$ have been reported before. No differences were observed between $O C T 1 * 2$ and $O C T 1 * 1$ in the uptake of $\mathrm{MPP}^{+}$[11], but strong differences were observed in the uptake of metformin, tropisetron or O-desmethyltramadol $[2,4,5]$. Substrate-specific differences were also observed for the Ser14Phe and Arg61Cys polymorphisms when the uptake of metformin and vitamin B1 (which behave fairly similarly) was compared with the $\mathrm{MPP}^{+}$uptake [1]. These substrate-specific effects were confirmed in the present study making artifacts from the cloning strategies or transport assays unlikely. Furthermore, our data shows that the substrate-specific effects are more general and are not restricted to the Met420del polymorphism and supports the existence of multiple binding sites that strongly vary among different OCT1 substrates. 
There are important practical consequences of the high percentage of polymorphisms with substrate specific effects on OCT1 activity. It is not sufficient to test the functional effects of a newly-identified amino acid substitution using only one substrate; a variant active with one substrate may be inactive with another substrate. Thus, the concept of using model OCT1 substrates especially for predicting effects of polymorphisms on the transport activity should be critically reevaluated. Our experiments showed only limited and strongly substrate-to-substrate variable ability of the model OCT1 substrates $\mathrm{MPP}^{+}, \mathrm{TEA}^{+}$, and $\mathrm{ASP}^{+}$to predict the effect of polymorphisms on the uptake of the clinically relevant drugs (Additional file 12). Therefore, at least until we do have more precise information about the different substrate binding mechanisms of OCT1, the effects of polymorphisms with known substrate-specific effects of OCT1 activity should be experimentally evaluated for each new OCT1 substrate.

\section{Conclusions}

We observed strong inter-regional variations in the frequency of loss of OCT1 activity. The differences in the frequencies of loss of OCT1 activity should be taken in consideration when transferring drug dosing recommendations from one population to another. This strong variability points the presence of an evolutionary selection for maintaining OCT1 activity in East Asian and Oceania, but the selection agent remains unknown. The major mechanistic cause of substrate-wide loss of OCT1 activity is improper membrane localization. However, a substantial number of the variants showed substratespecific loss of activity. This reflects the poly-specificity of the OCT1 transporter and suggests different substrate binding sites and transport mechanisms for the different OCT1 substrates.

\section{Additional files}

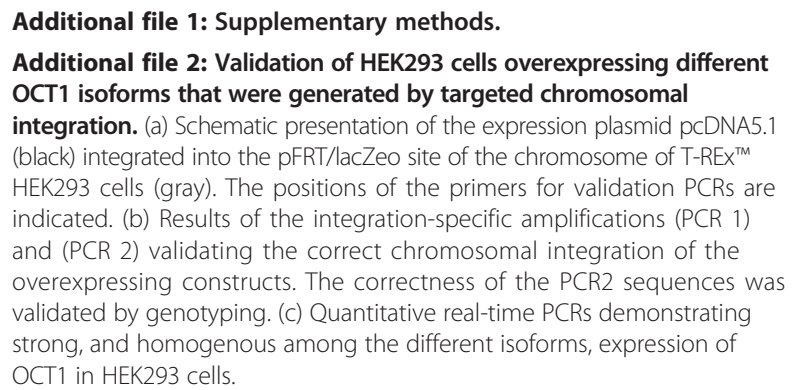

Additional file 3: List of the different tools used for prediction of the effects of $O C T 1$ polymorphisms on protein function.

Additional file 4: Re-sequencing analyses of OCT1. (a) Size of the re-sequenced regions stratified according their function: CDS, (protein) coding sequence; UTR, untranslated region, and intron-exon borders. (b) List of the 11 re-sequenced regions defined with their absolute and relative positions at chromosome 6. (c) Schematic representation of the targeted re-sequencing regions and an example of the sequencing coverage. The amplicons from the two multiplex reactions used are indicated in blue and red color. For details see the Materials and methods section.

Additional file 5: A detailed list of genetic variants identified by massively parallel sequencing of the enriched HGDP-CEPH samples. Additional file 6: In silico prediction of functional impact of amino acid substitutions observed in the worldwide genetic analyses of OCT1.

Additional file 7: Evolutionary conservation of the variable OCT1 sites in OCT1 orthologs (upper part) and paralogs (lower part).

Additional file 8: A detailed worldwide distribution of $O C T 1$ alleles including the distribution of the sub-alleles.

Additional file 9: Characterization of tyramine uptake in HEK 293 cells overexpressing OCT1. (a) Tyramine inhibits the uptake of $\mathrm{MPP}^{+}$. The cellular accumulation of 1-methyl-4-phenylpyridinium $\left(\mathrm{MPP}^{+}\right)$was measured after 1 min exposure to $10 \mathrm{nM}^{3} \mathrm{H} \mathrm{MPP}^{+}$in cells overexpressing $\mathrm{OCT} 1{ }^{*} 1$. The graph shows mean values and standard errors of the mean from three independent experiments. (b) Concentration dependence of tyramine uptake. OCT1*1-overexpressing and control cells (stably transfected with the empty pcDNA5.1/FRT plasmid were incubated for

1 min with increasing concentrations of tyramine. The graphs show mean values and standard errors of the mean of three independent experiments. (c) The OCT1-mediated uptake. The OCT1-mediated fraction of the total uptake was calculated by subtracting the uptake in the control cells from the uptake in OCT1*1-overexpressing cells using the dataset shown in (b).

Additional file 10: Differences in the uptake between $\mathrm{OCT} 1 * 1$ overexpressing (WT) and control cells stably transfected with the empty pcDNA5.1/FRT plasmid (pcDNA5). The graphs show mean values and standard errors of the mean of three or more independent experiments.

Additional file 11: Lack of interaction between Met408Val and Met420del polymorphisms in the OCT1 gene. (a) Comparison of the uptake activity of OCT1 carrying all theoretically possible combinations of the Met420del and Met408Val polymorphisms. The uptake was measured in HEK293 cells stably transfected with the OCT1 variants or with an empty control plasmid pCDNA5. Shown are means and standard error of the means of at least three independent experiments. (b) Subcellular localization of OCT1 isoforms carrying the four different combination of the Met420del and Met408Val polymorphisms. The protein is located on the plasma membrane indicated by co-staining with anti- $\mathrm{Na}^{+} / \mathrm{K}^{+}$ATPase as a plasma membrane marker. No qualitative differences could be observed between cells carrying Met408 or Val408 independent from their background (Met420 or Del420). (c) Frequencies of the different single polymorphism genotypes (the upper part) and haplotype combinations (the lower part) in the HGDP-CEPH sample. Individual haplotypes were inferred using PHASE version 2.1. The genotype/ haplotype frequencies are given as number (and \%) of all individuals.

Additional file 12: Correlation between the effects of substratespecific loss of function OCT1 alleles on the transport of model substrates and clinically relevant drugs. We analyzed correlations between the uptake of model OCT1 substrates (MPP+ ${ }^{+}, \mathrm{TEA}^{+}, \mathrm{ASP}^{+}$) and clinically relevant drugs (metformin and morphine) for all the OCT1 alleles that show substrate-specific loss of activity, that is, ${ }^{*} 2,{ }^{*} 7$, ${ }^{*} 10, * 11$, and ${ }^{*} 13$. Shown are means and standard error of the means of at least three independent transport experiments. Solid lines represent linear regression, dashed lines represent an optimal theoretical correlation with identical effects of the loss-of-function allele on both the model substrates and drug. ${ }^{*}$ denotes significant correlations with $P<0.05$.

Additional file 13: Correct subcellular localization of OCT1 alleles ${ }^{*} \mathbf{1},{ }^{2} \mathbf{2} * \mathbf{8}, * \mathbf{9},{ }^{*} \mathbf{1 0}, * \mathbf{1 1}$, and ${ }^{*} \mathbf{1 3}$. The analyzed alleles are known to have normal $\left(O C T 1{ }^{*} 1,{ }^{*} 1 B,{ }^{*} 1 C\right.$, and $\left.{ }^{*} 1 D\right)$, increased $\left(O C T 1 * 8\right.$ and $\left.{ }^{*}\right)$ ) or substrate-specific loss of OCT1 activity $\left({ }^{*} 2,{ }^{*} 10,{ }^{*} 11\right.$, and $\left.{ }^{*} 13\right)$. The subcellular localization of the different OCT1 isoforms was analyzed after immunocytochemical staining of OCT1 (green) in combination 
with $\mathrm{Na}^{+} / \mathrm{K}^{+}$ATPase (red, upper part) as a marker for plasma membrane and calnexin (red, lower part) as a marker for endoplasmic reticulum.

Additional file 14: Detailed maps of the interpopulation variation of the frequency of loss of OCT1 activity in Europe (a) and China (b). Additional file 15: Pairwise analyses of divergence based on the frequencies of OCT1 alleles in populations and in different world regions (the upper panel). The deep blue color correspond to high divergence between the populations / regions (high $\mathrm{F}_{\mathrm{ST}}$ values). We analyzed 62 population from the 1000 Genomes Project (designated with $1 \mathrm{~K}$ ) and the HGDP-CEPH project (the rest). The populations were stratified in world regions as listed in Table 1. In contrast to Fig. 7, which shows divergence at the level of loss of OCT1 activity, this figure shows divergences at the level of single OCT1 alleles.

\section{Competing interests}

The authors declare that they have no competing interests.

\section{Authors' contributions}

TS, RS, JC, and JDSP performed the experiments and analyzed the data. SP performed the experiments. ND and RK analyzed the data. JB wrote the manuscript. MVT planned the study, analyzed the data, and wrote the manuscript. All authors read and approved the final manuscript.

\section{Acknowledgments}

We are highly grateful to Howard Cann and his colleagues for enable us using the HGDP-CEPH DNA bank, which was essential for performing this study. We would like to acknowledge Karoline Jobst for the excellent technical support and Valerie O'Brien for the interesting discussions.

This study has been financially supported by the German Research Foundation (DFG) grants GRK1034, TZ 74/1-1 and the Clinical Research Group 'Genotype-phenotype relationships and neurobiology of the longitudinal course of psychosis' in work package 3 (http://www.kfo241.de; grant number BR 2471/1-1), to JB and MVT.

\section{Author details}

${ }^{1}$ Institute of Clinical Pharmacology, University Medical Center Göttingen, Robert-Koch-Str. 40, DE-37075 Göttingen, Germany. ${ }^{2}$ Department of Pediatrics and Adolescent Medicine, University Medical Center Göttingen, Robert-Koch-Str. 40, DE-37075 Göttingen, Germany.

Received: 10 March 2015 Accepted: 11 May 2015

Published online: 18 June 2015

\section{References}

1. Chen L, Shu Y, Liang X, Chen EC, Yee SW, Zur AA, et al. OCT1 is a high-capacity thiamine transporter that regulates hepatic steatosis and is a target of metformin. Proc Natl Acad Sci U S A. 2014;111:9983-8.

2. Shu Y, Sheardown SA, Brown C, Owen RP, Zhang S, Castro RA, et al. Effect of genetic variation in the organic cation transporter 1 (OCT1) on metformin action. J Clin Invest. 2007;117:1422-31.

3. Tzvetkov MV, dos Santos Pereira JN, Meineke I, Saadatmand AR, Stingl JC, Brockmoller J. Morphine is a substrate of the organic cation transporter OCT1 and polymorphisms in OCT1 gene affect morphine pharmacokinetics after codeine administration. Biochem Pharmacol. 2013;86:666-78.

4. Tzvetkov MV, Saadatmand AR, Bokelmann K, Meineke I, Kaiser R, Brockmoller J. Effects of OCT1 polymorphisms on the cellular uptake, plasma concentrations and efficacy of the $5-\mathrm{HT}(3)$ antagonists tropisetron and ondansetron. Pharmacogenomics J. 2012;12:22-9.

5. Tzvetkov MV, Saadatmand AR, Lotsch J, Tegeder I, Stingl JC, Brockmoller J. Genetically polymorphic OCT1: another piece in the puzzle of the variable pharmacokinetics and pharmacodynamics of the opioidergic drug tramadol. Clin Pharmacol Ther. 2011;90:143-50.

6. Zhang L, Dresser MJ, Gray AT, Yost SC, Terashita S, Giacomini KM. Cloning and functional expression of a human liver organic cation transporter. Mol Pharmacol. 1997;51:913-21.

7. Herraez E, Lozano E, Macias RI, Vaquero J, Bujanda L, Banales JM, et al. Expression of SLC22A1 variants may affect the response of hepatocellular carcinoma and cholangiocarcinoma to sorafenib. Hepatology. 2013;58:1065-73
8. Nies AT, Koepsell H, Winter S, Burk O, Klein K, Kerb R, et al. Expression of organic cation transporters OCT1 (SLC22A1) and OCT3 (SLC22A3) is affected by genetic factors and cholestasis in human liver. Hepatology. 2009;50:1227-40.

9. Tu M, Sun S, Wang K, Peng X, Wang R, Li L, et al. Organic cation transporter 1 mediates the uptake of monocrotaline and plays an important role in its hepatotoxicity. Toxicology. 2013;311:225-30.

10. Kerb R, Brinkmann U, Chatskaia N, Gorbunov D, Gorboulev V, Mornhinweg E, et al. Identification of genetic variations of the human organic cation transporter hOCT1 and their functional consequences. Pharmacogenetics. 2002;12:591-5.

11. Shu Y, Leabman MK, Feng B, Mangravite LM, Huang CC, Stryke D, et al. Evolutionary conservation predicts function of variants of the human organic cation transporter, OCT1. Proc Natl Acad Sci U S A. 2003;100:5902-7.

12. Fukuda $T$, Chidambaran $V$, Mizuno $T$, Venkatasubramanian $R$, Ngamprasertwong P, Olbrecht $V$, et al. OCT1 genetic variants influence the pharmacokinetics of morphine in children. Pharmacogenomics. 2013;14:1141-51.

13. Nies A, Koepsell H, Damme K, Schwab M. Organic cation transporters (OCTs, MATEs), In Vitro and In Vivo evidence for the importance in drug therapy. Handb Exp Pharmacol. 2011;201:105-67.

14. Jonker JW, Wagenaar E, Mol CA, Buitelaar M, Koepsell H, Smit JW, et al. Reduced hepatic uptake and intestinal excretion of organic cations in mice with a targeted disruption of the organic cation transporter 1 (Oct1 [Slc22a1]) gene. Mol Cell Biol. 2001;21:5471-7.

15. Wang DS, Jonker JW, Kato $Y$, Kusuhara H, Schinkel AH, Sugiyama $Y$. Involvement of organic cation transporter 1 in hepatic and intestinal distribution of metformin. J Pharmacol Exp Ther. 2002;302:510-5.

16. Leabman MK, Huang CC, DeYoung J, Carlson EJ, Taylor TR, de la Cruz M, et al. Natural variation in human membrane transporter genes reveals evolutionary and functional constraints. Proc Natl Acad Sci U S A. 2003;100:5896-901.

17. Chen L, Takizawa M, Chen E, Schlessinger A, Segenthelar J, Choi JH, et al. Genetic polymorphisms in organic cation transporter 1 (OCT1) in Chinese and Japanese populations exhibit altered function. J Pharmacol Exp Ther. 2010;335:42-50.

18. Itoda M, Saito Y, Maekawa K, Hichiya H, Komamura K, Kamakura S, et al. Seven novel single nucleotide polymorphisms in the human SLC22A1 gene encoding organic cation transporter 1 (OCT1). Drug Metab Pharmacokinet. 2004;19:308-12.

19. Saito S, lida A, Sekine A, Ogawa C, Kawauchi S, Higuchi S, et al. Catalog of 238 variations among six human genes encoding solute carriers (hSLCS) in the Japanese population. J Hum Genet. 2002;47:576-84.

20. Sakata T, Anzai N, Shin HJ, Noshiro R, Hirata T, Yokoyama H, et al. Novel single nucleotide polymorphisms of organic cation transporter 1 (SLC22A1) affecting transport functions. Biochem Biophys Res Commun. 2004;313:789-93.

21. Takeuchi A, Motohashi H, Okuda M, Inui K. Decreased function of genetic variants, Pro283Leu and Arg287Gly, in human organic cation transporter hOCT1. Drug Metab Pharmacokinet. 2003;18:409-12.

22. Cann HM, de Toma C, Cazes L, Legrand MF, Morel V, Piouffre L, et al. A human genome diversity cell line panel. Science. 2002;296:261-2.

23. Tzvetkov MV, Vormfelde SV, Balen D, Meineke I, Schmidt T, Sehrt D, et al. The effects of genetic polymorphisms in the organic cation transporters OCT1, OCT2, and OCT3 on the renal clearance of metformin. Clin Pharmacol Ther. 2009;86:299-306.

24. 1000 Genomes Project Consortium, Abecasis GR, Auton A, Brooks LD, DePristo MA, Durbin RM, et al. An integrated map of genetic variation from 1,092 human genomes. Nature. 2012;491:56-65.

25. Saadatmand AR, Tadjerpisheh S, Brockmoller J, Tzvetkov MV. The prototypic pharmacogenetic drug debrisoquine is a substrate of the genetically polymorphic organic cation transporter OCT1. Biochem Pharmacol. 2012;83:1427-34.

26. Stephens M, Smith NJ, Donnelly P. A new statistical method for haplotype reconstruction from population data. Am J Hum Genet. 2001;68:978-89.

27. Excoffier L, Laval G, Schneider S. Arlequin (version 3.0): an integrated software package for population genetics data analysis. Evol Bioinform Online. 2005;1:47-50

28. HGDP-CEPH Database. Available at: http://www.cephb.fr/en/hgdp_panel.php. 
29. Schomig E, Lazar A, Grundemann D. Extraneuronal monoamine transporter and organic cation transporters 1 and 2: a review of transport efficiency. Handb Exp Pharmacol. 2006;175:151-80.

30. Pasanen MK, Neuvonen PJ, Niemi M. Global analysis of genetic variation in SLCO1B1. Pharmacogenomics. 2008;9:19-33.

31. Sistonen J, Sajantila A, Lao O, Corander J, Barbujani G, Fuselli S. CYP2D6 worldwide genetic variation shows high frequency of altered activity variants and no continental structure. Pharmacogenet Genomics. 2007;17:93-101.

32. Niemi M. Transporter pharmacogenetics and statin toxicity. Clin Pharmacol Ther. 2010;87:130-3.

33. Niemi M, Pasanen MK, Neuvonen PJ. Organic anion transporting polypeptide 1B1: a genetically polymorphic transporter of major importance for hepatic drug uptake. Pharmacol Rev. 2011;63:157-81.

34. Group SC, Link E, Parish S, Armitage J, Bowman L, Heath S, et al. SLCO1B1 variants and statin-induced myopathy-a genomewide study. N Engl J Med. 2008:359:789-99.

35. Urban TJ, Sebro R, Hurowitz EH, Leabman MK, Badagnani I, Lagpacan LL, et al. Functional genomics of membrane transporters in human populations. Genome Res. 2006;16:223-30.

36. Sadhasivam S, Krekels EH, Chidambaran V, Esslinger HR, Ngamprasertwong $P$, Zhang $\mathrm{K}$, et al. Morphine clearance in children: does race or genetics matter? J Opioid Manag. 2012:8:217-26.

37. Li JZ, Absher DM, Tang H, Southwick AM, Casto AM, Ramachandran S, et al. Worldwide human relationships inferred from genome-wide patterns of variation. Science. 2008;319:1100-4.

38. Nebert DW. Polymorphisms in drug-metabolizing enzymes: what is their clinical relevance and why do they exist? Am J Hum Genet. 1997;60:265-71.

39. Masson F, Talon R, Montel MC. Histamine and tyramine production by bacteria from meat products. Int J Food Microbiol. 1996;32:199-207.

40. Pechanek U, Pfannhauser W, Woidich H. Untersuchung fiber den Gehalt biogener Amine in vier Gruppen von Lebensmitteln des iisterreichischen Marktes. Zeitschift for Lebensmittel Untersuchung und Forschung. 1983;176:335-40.

41. Grimsby J, Lan NC, Neve R, Chen K, Shih JC. Tissue distribution of human monoamine oxidase A and B mRNA. J Neurochem. 1990;55:1166-9.

42. Bf R. Pyrrolizidinalkaloide in Kräutertees und Tees 018/2013 edition. Berlin: BfR - Bundesinstitut für Risikobewertung; 2013.

43. Fu PP, Xia Q, Lin G, Chou MW. Pyrrolizidine alkaloids-genotoxicity, metabolism enzymes, metabolic activation, and mechanisms. Drug Metab Rev. 2004;36:1-55.

44. Worldwide distribution map of Crotalaria retusa (rattleweed). Available at: www.cabi.org/isc/datasheet/87408.

45. Li L, Tu M, Yang X, Sun S, Wu X, Zhou H, et al. The contribution of human OCT1, OCT3, and CYP3A4 to nitidine chloride-induced hepatocellular toxicity. Drug Metab Dispos. 2014;42:1227-34.

46. Tu M, Li L, Lei H, Ma Z, Chen Z, Sun S, et al. Involvement of organic cation transporter 1 and CYP3A4 in retrorsine-induced toxicity. Toxicology. 2014;322:34-42.

47. Liu H, Prugnolle F, Manica A, Balloux F. A geographically explicit genetic model of worldwide human-settlement history. Am J Hum Genet. 2006:79:230-7.

48. Lourenco AE, Santos RV, Orellana JD, Coimbra Jr CE. Nutrition transition in Amazonia: obesity and socioeconomic change in the Surui Indians from Brazil. Am J Hum Biol. 2008;20:564-71.

49. Keller T, Egenberger B, Gorboulev V, Bernhard F, Uzelac Z, Gorbunov D, et al. The large extracellular loop of organic cation transporter 1 influences substrate affinity and is pivotal for oligomerization. J Biol Chem. 2011;286:37874-86

50. Koepsell H. Substrate recognition and translocation by polyspecific organic cation transporters. Biol Chem. 2011;392:95-101.

51. ModBase: database of comparative protein structure models. Available at: http://modbase.compbio.ucsf.edu.

\section{Submit your next manuscript to BioMed Central and take full advantage of:}

- Convenient online submission

- Thorough peer review

- No space constraints or color figure charges

- Immediate publication on acceptance

- Inclusion in PubMed, CAS, Scopus and Google Scholar

- Research which is freely available for redistribution

Submit your manuscript at www.biomedcentral.com/submit 